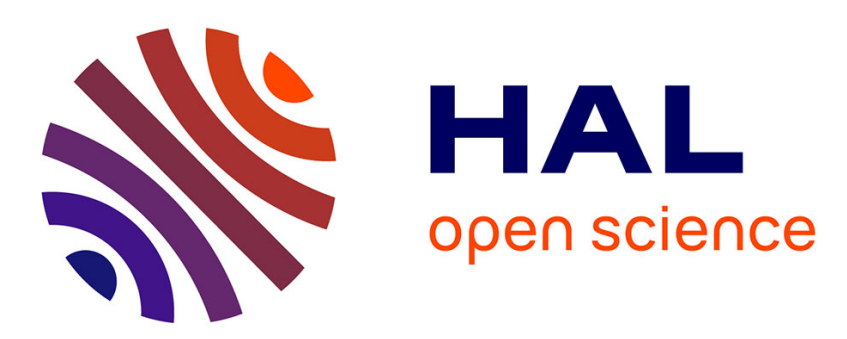

\title{
Characterization of microcrystalline cellulose spheres and prediction of hopper flow based on a $\mu(\mathrm{I})$-rheology model.
}

Johan Remmelgas, Abdoulaye Fall, Srdjan Sasic, Henrik Ström, Pirjo Tajarobi, Håkan Wikström, Mariagrazia Marucci, Catherine Boissier

\section{To cite this version:}

Johan Remmelgas, Abdoulaye Fall, Srdjan Sasic, Henrik Ström, Pirjo Tajarobi, et al.. Characterization of microcrystalline cellulose spheres and prediction of hopper flow based on a $\mu(\mathrm{I})$-rheology model.. European Journal of Pharmaceutical Sciences, 2019, pp.105085. 10.1016/j.ejps.2019.105085 . hal02346496

\section{HAL Id: hal-02346496 \\ https://hal.science/hal-02346496}

Submitted on 5 Nov 2019

HAL is a multi-disciplinary open access archive for the deposit and dissemination of scientific research documents, whether they are published or not. The documents may come from teaching and research institutions in France or abroad, or from public or private research centers.
L'archive ouverte pluridisciplinaire HAL, est destinée au dépôt et à la diffusion de documents scientifiques de niveau recherche, publiés ou non, émanant des établissements d'enseignement et de recherche français ou étrangers, des laboratoires publics ou privés. 
1 Characterization of microcrystalline cellulose spheres and prediction of hopper

2 flow based on a $\mu(I)$-rheology model.

3

4

5

6

7

8

Johan Remmelgas ${ }^{a}{ }^{a}$, Abdoulaye Fall ${ }^{b}$, Srdjan Sasic ${ }^{c}$, Henrik Ström ${ }^{c}$, Pirjo Tajarobi ${ }^{d}$, Håkan $^{\circ}$ Wikström $^{\mathrm{a}}$, Mariagrazia Marucci ${ }^{\mathrm{a}}$, Catherine Boissier ${ }^{\mathrm{d}}$.

a Pharmaceutical Technology \& Development, AstraZeneca, Gothenburg, Sweden.

${ }^{\mathrm{b}}$ Université Paris Est, Laboratoire Navier (UMR 8205 CNRS, IFSTTAR, Ecole des Ponts ParisTech), Champs-sur-Marne, France.

${ }^{\mathrm{c}}$ Chalmers University of Technology, Department of Mechanics and Maritime Sciences, Gothenburg, Sweden.

d Pharmaceutical Sciences, IMED Biotech Unit, AstraZeneca, Gothenburg, Sweden.

\section{Abstract}

The objective of this study was to characterize the rheology of a pharmaceutical material in the context of the $\mu(I)$-rheology model and to use this model to predict powder flow in a manufacturing operation that is relevant to pharmaceutical manufacturing. The rheology of microcrystalline cellulose spheres was therefore characterized in terms of the $\mu(I)$-rheology model using a modified Malvern Kinexus rheometer. As an example of an important problem in pharmaceutical manufacturing, the flow of these particles from a hopper was studied experimentally and numerically using a continuum Navier-Stokes solver based on the Volume-Of-Fluid (VOF) interface-capturing numerical method. The work shows that the rheology of this typical pharmaceutical material can be measured using a modified annular shear rheometer and that the results can be interpreted in terms of the $\mu(I)$-rheology model. It is demonstrated that both the simulation results and the experimental data show a constant hopper discharge rate. It is noted that the model can suffer from ill-posedness and it is shown how an increasingly fine grid resolution can result in predictions that are not entirely physically realistic. This shortcoming of the numerical framework implies that caution is required when making a one-to-one comparison with experimental data. 
31 Keywords: Powder; Hopper; Rheology; Inertial number; Volume-Of-Fluid

32

$33{ }^{*}$ Corresponding author. AstraZeneca, 43183 Mölndal, Sweden. Tel.: +46 722 349420. E-mail 34 address: johan.remmelgas@astrazeneca.com (J. Remmelgas). 


\section{Introduction}

Flow and discharge of powders in process vessels are well known to give rise to significant handling problems in a range of processes, for example in the pharmaceutical, mineral, chemical and food industries. Common problems include ratholing, arching in silos during discharge, blockage of pipes during powder transport, and agglomeration or caking. While predictive tools for powder flow are available in special cases, a quantitative description of powder flow that is generally applicable is still missing. The current understanding of the different flow regimes in such systems is therefore largely empirical as the granular state is rather difficult to characterize, it being neither liquid nor solid and sharing some aspects of both (Campbell, 2006).

When modeling powder flow, one may choose to view the powder as a solid or a fluid and thus use methods based on either solid or fluid mechanics. A considerable amount of work has been carried out to quantitatively predict powder flow in, for example, hoppers and blenders (Zheng and Yu, 2015a, 2015b; Zheng et al., 2017a, 2017b; Bai et al., 2017; Liu et al., 2018a, 2018b) using methods that have been developed from a solid mechanics background. A comparable amount of work has also been carried out using the discrete element method (see e.g. Anand et al., 2008, 2009; Ketterhagen et al. 2009; Persson et al., 2011), which is conceptually straightforward and therefore very attractive. However, discrete element methods are usually difficult to apply to systems of interest in the pharmaceutical industry since the number of particles for these systems is very large and the associated computational effort then is too high. To this end, hybrid methods such as the dense discrete phase model (Popoff and Braun, 2007; Chen and Wang, 2014), in which particles are still tracked in a Lagrangian framework, but their collisions modelled using concepts from the Kinetic Theory of Granular Flow, have been introduced in a number of applications. In addition, coarse-grained discrete element methods have been used to simulate systems of many small particles replacing a group of original particles by a significantly larger (pseudo) particle (Bierwish et al., 2009; Queteschiner et al., 2018).

We are ultimately interested in modeling the flow of powders in processes that are relevant to commercial pharmaceutical manufacturing operations. Since these operations involve large quantities of powders with a particle size that is on the order of 100 micron, some form of continuum model is preferred. For particles in this size range aerodynamic forces play an 
important role and it is thus natural to select a modeling approach that is based upon a fluid mechanics description of powder flow.

Recently, there has been renewed interest in developing a predictive capability for powder or granular flows. This research has to a great extent relied on numerical simulations of systems with small numbers of particles using discrete element methods (e.g. Chialvo et al., 2012; da Cruz et al., 2005; Hatano, 2007) to formulate continuum-level constitutive relations for powders (Jop et al., 2006). In particular, the model of Jop et al. (2006), which has become known as the $\mu(I)$-rheology model, has been used to correctly predict velocity profiles in complex three-dimensional flows (Jop et al. 2006). The $\mu(I)$-rheology model has since been generalized (Chialvo et al., 2012) and successfully used in a nontrivial powder mechanics context to describe situations ranging from the collapse of a granular column (Dunatunga and Kamrin, 2015) to the flow in bubbling fluidized beds (Farzaneh et al., 2015; Schneiderbauer et al., 2012). The $\mu(I)$-rheology model has also been extended to include cohesive powders (e.g. Berger et al., 2015; Khamseh et al., 2015; Rognon et al., 2008) and effects of non-local momentum transfer (Kamrin and Koval, 2012; Henann and Kamrin, 2013). The latter extension to include non-local rheology is noteworthy since it represents the first time that a continuum model was used to correctly predict the flow pattern in a split-bottom annular shear cell (Henann and Kamrin, 2013).

In the $\mu(I)$-rheology model, which was developed for simple granular materials composed of nearly identical spherical particles interacting only via dry solid contacts, the tangential stress is proportional to the normal stress via the effective friction coefficient via

$\tau=\mu(I) P$

where $\tau$ is the shear stress, $P$ is the normal stress, and $\mu(I)$ is the effective friction coefficient, which depends on the inertial number, $I$. The inertial number is defined as

$I=\frac{\dot{\gamma} d}{\sqrt{P / \rho}}$ where $\dot{\gamma}$ is the shear rate, $d$ is the particle diameter, and $\rho$ is the particle density. Several suggestions for the dependence of the effective friction coefficient on the inertial number 
have been made, including the one by Jop et al. (2006) in which the effective friction coefficient is expressed as

$\mu(I)=\mu_{s}+\frac{\mu_{2}-\mu_{s}}{\frac{I_{0}}{I}+1}$

where $\mu_{s}$ and $\mu_{2}$ are, respectively, the friction coefficients at very low and very high inertial numbers and $I_{0}$ is a constant. The $\mu(I)$-rheology model does not explicitly include material elasticity or plasticity or the effects of a particle size or shape distribution. Instead, all aspects of the material are included in the model parameters $\mu_{s}, \mu_{2}$, and $I_{0}$, which thus can be expected to be material-dependent. The fact that the model can be used to describe granular flows using only a few parameters that are, at least conceptually, straightforward to measure in an independent experiment obviously makes it quite attractive.

The inertial number can be understood as a dimensionless shear rate that expresses the relationship between the time scale for particle rearrangements due to the confining pressure and the time scale for deformation by the mean flow (GDR Midi, 2004). The inertial number thus characterizes the local rapidity of the flow and allows for a classification of flow regimes. As the inertial number increases, the average contact time decreases and the material dilates. For $l<0.01$, the material is in the quasi-static regime, in which it can be described as an elasto-plastic solid (Andreotti et al., 2013) while for $I>0.1$ the material approaches the collisional regime that can be described by the kinetic theory of granular flow (Campbell, 1990). The intermediate regime $(0.01<I<0.1)$ corresponds to dense flows where the material is above its flow threshold but in a liquid-like rather than gas-like state. One may also view the inertial number as the ratio of collisional to total stress. As an alternative to the inertial number, one may characterize this ratio using the Savage number (Savage and Hutter, 1989) or the Coulomb number (Ancey et al., 1999), both of which are the square of the inertial number.

The introduction of the $\mu(I)$-rheology model was a breakthrough in the effort to develop a continuum-level framework aimed at describing the wide variety of behaviors that granular flows can exhibit. Despite the importance of powder flow predictions in pharmaceutical manufacturing, no powder that is of interest in the pharmaceutical industry has been characterized in the context of the $\mu(I)$-rheology model. The objective of this study is therefore to characterize a pharmaceutical material in the context of this model and to use 
the resulting characterization to simulate a flow problem that is important in pharmaceutical manufacturing. We therefore characterize VIVAPUR MCC Spheres 500, a material that is of great value in the pharmaceutical industry (for e.g. multiple-unit pellet systems), to obtain model parameters for the $\mu(I)$-rheology model. We then carry out hydrodynamic predictions based upon this model using the interface-capturing Volume-Of-Fluid (VOF) numerical framework for the important case of hopper discharge. The results from the simulations are compared with experimental data on the same system. Although the VIVAPUR MCC Spheres 500 have a particle size that is larger than most pharmaceutical excipients, it is a suitable material for this initial work since the particle size is large enough that aerodynamic effects can be expected to be small compared to the frictional effects that are described by the $\mu(I)$-rheology model.

\section{Material and methods}

\subsection{Material characterization}

The material used in this study is microcrystalline cellulose spheres (VIVAPUR MCC Spheres 500, JRS PHARMA GmbH \& Co. KG, Germany). The bulk density was determined according to Method 1 of the European Pharmacopoeia (2018).

SEM images were obtained using a Hitachi TM3030Plus Tabletop microscope (Hitachi HighTechnologies Corporation, Ichiga, Japan). The particle size and shape distribution were determined using a Camsizer X2 optical particle size and shape analyzer (Retsch Technology, Haan, Germany). As measures of particle shape, the sphericity or circularity (Cox, 1927) and the aspect ratio are used.

\subsection{Steady shear experiments under confinement pressure}

The flow properties of the MCC spheres were experimentally characterized using a modified Malvern Kinexus rheometer (see Figure 1) following the procedure suggested by Fall et al. (2015). In this procedure, the sample is poured and compacted by repeatedly tapping it in 
an annular shear cell with inner and outer radii of 21 and $45 \mathrm{~mm}$. The filling height of the annulus is adjustable from approximately $3 \mathrm{~mm}$ to approximately $25 \mathrm{~mm}$.

\section{RHEOMETER}

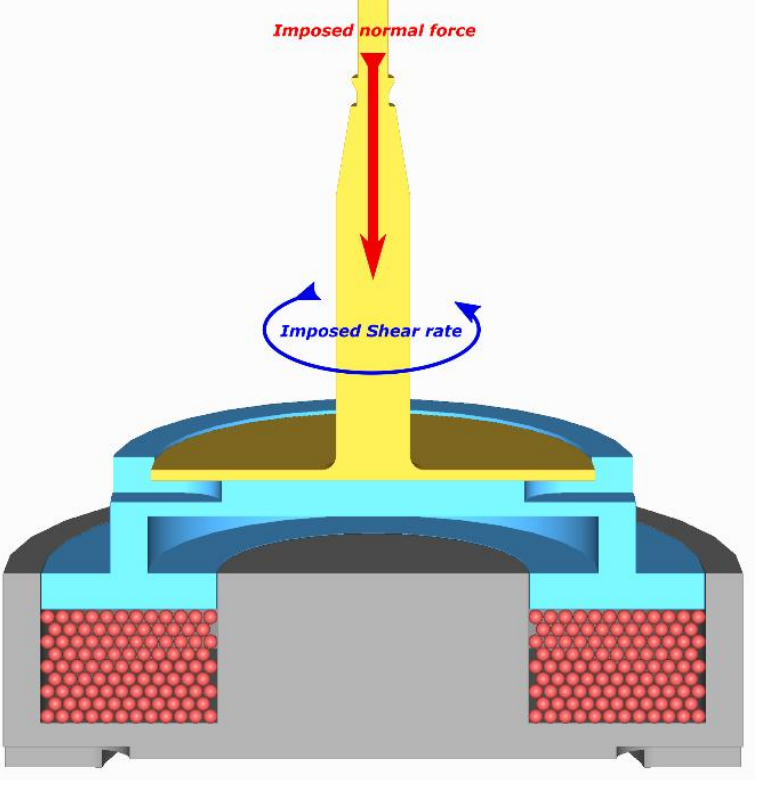

Figure 1: Sketch of a modified Malvern Kinexus rheometer used in this work for characterizing pharmaceutical powders (Fall et al., 2015).

This normal stress-controlled annular apparatus is designed to carry out experiments in which the granular phase dilates or contracts, depending on the density necessary to support the confining pressure under the imposed shear rate. This gives instantaneous measurements of the driving shear stress and the gap for an imposed normal force and shear rate. The solid volume fraction is not fixed but adjusts to the imposed shear rate. Since the particles cannot escape from the shear cell, the solid volume can be measured. Instead of setting the gap size for a given experiment, a normal force (i.e. the normal confining stress) is imposed. While shearing, the gap size is then varied in order to maintain the desired value of the normal force.

In this work, experiments were carried out at room temperature $\left(20^{\circ} \mathrm{C}\right)$ in an environment with the relative humidity of approximately $55 \%$. After pouring the sample into the annular shear cell, the samples were left to equilibrate in this environment for approximately 2 hours before carrying out the experiments. A normal force of $2 \mathrm{~N}$ was applied to generate a confining normal pressure of $400 \mathrm{~Pa}$ and shear rates between 0.002 to $95 \mathrm{~s}^{-1}$ were then 
explored. No discernible change in the particle morphology was observed as a result of this experimental characterization.

\subsection{Determination of model parameters}

The parameters $\mu_{s}, \mu_{2}$, and $I_{0}$ are obtained by fitting the effective friction coefficient in Equation (3) to the experimentally determined friction coefficient using a classical least squares method for the range of inertial numbers that were explored. Although not used in the prediction of hopper discharge in this work, the dependence of the solids volume fraction on the inertial number is also characterized. The dependence of the solids volume fraction on the inertial number is modeled using the model suggested by da Cruz et al. (2005),

$\phi=\phi_{\max }-\left(\phi_{\max }-\phi_{\min }\right) I$

where $\phi$ is the solid volume fraction and $\phi_{\max }$ and $\phi_{\min }$ are model parameters. As for the other model parameters, $\phi_{\max }$ and $\phi_{\min }$ are determined by fitting Equation (4) to experimental data using a classical least squares method for the range of inertial numbers that were explored.

\subsection{Experimental evaluation of flow of MCC spheres from a hopper}

The flow behavior of the VIVAPUR MCC spheres 500 in a hopper was studied experimentally using a unit with the same dimensions as the one used for testing sifting segregation described in ASTM D6940-03 (2003). As shown in Figure 2 (top), this unit features a hopper with an upper cylindrical part with a height of $127 \mathrm{~mm}$ and a converging section with a height of $36 \mathrm{~mm}$. The diameter of the upper cylindrical part is $D_{c}=127 \mathrm{~mm}$, while the diameter of the hopper outlet is $D_{o}=25 \mathrm{~mm}$.

In the experiments, $1000 \mathrm{~g}$ of MCC spheres was filled into the hopper. At a certain point in time the hopper outlet was opened and the powder allowed to flow out into a bowl that was placed on a balance (K-SFS-24I/24N, Coperion K-Tron, Niederlenz, Switzerland). It was assumed that the weight recorded by the balance (K-Sampler, Coperion K-Tron, Niederlenz, 
Switzerland) at any point in time corresponded to the amount of powder in the bowl. In this manner, the discharged mass as a function of time was measured. While the balance reports the weight only one time per second, the reported weight is actually the average of approximately 200 measurements during that second.

\subsection{Selection of confinement pressure for steady shear experiments}

For the present hopper, the MCC spheres are expected to exhibit funnel flow and the stress in the converging section of the hopper can therefore not be estimated using Jenike's methodology for mass flow bins (Jenike, 1961). Instead, we therefore estimate the stress in the upper cylindrical part of the hopper using Janssen's equation (Janssen, 1895), which may be expressed

$P_{v}=\frac{g \rho_{0} D_{c}}{4 k_{J} \mu_{W}}\left[1-e^{-4 k_{J} \mu_{W} z / D_{c}}\right]$

where $P_{v}$ is the vertical pressure, $\rho_{0}$ is the bulk density, $g=9.81 \mathrm{~m} / \mathrm{s}^{2}$ is the acceleration due to gravity, $D_{c}$ is the diameter of the upper cylindrical part of the hopper, $k_{\jmath}$ is the stress ratio, $\mu_{W}$ is the coefficient of wall friction, and $z$ is the distance below the surface of the powder bed. For the purposes of this work, it is assumed that $k_{J}=0.4$ since this value is applicable to many materials (Jenike et al., 1973).

\subsection{Prediction of discharge rate using Beverloo correlation}

For particles that are large enough that aerodynamic drag is not significant, the Beverloo correlation (Beverloo et al., 1961) can be used to predict the discharge rate. The Beverloo correlation is an almost universal characteristic for gravity flow of granular materials through orifices. It may be written

$\dot{m}=C \rho_{0} \sqrt{g}\left(D_{o}-k d\right)^{5 / 2}$

where $\dot{m}$ is the mass flow rate, $D_{o}$ is diameter of the hopper outlet, and $C$ and $k$ are constants. It has been found that $C$ is approximately 0.58 (Nedderman et al., 1982) while $k$ depends on the material and is between approximately 1 and 3 (Spink and Nedderman, 1978). 


\subsection{Simulation of hopper discharge using the $\mu(I)$-rheology model}

Since the hopper outlet is much larger than the particle diameter, continuum modelling of the discharge process is justifiable. As in the work of Lagrée et al. (2011) and Staron et al. (2012, 2014), continuum simulations using a Volume-Of-Fluid framework (VOF) are carried out. The VOF method is a single-fluid framework in which the actual presence of two fluids is modeled by introducing the volume fraction of each component and a procedure to track the interface between the components. That is, in the VOF method the location of the interface between the components is not imposed but rather captured by solving its transport equation. In this case, the first fluid component is the MCC powder (including the interstitial air) while the other one is the surrounding air. Although the air has no or almost no effect on the powder phase for the present MCC spheres, it has to be included because something must occupy the part of the domain where there are no particles. The constitutive behavior of the MCC powder is characterized by a $\mu(I)$-rheology model. Note that the mass and momentum conservation equations to be solved within the framework are not given here, since they have the same structure as the Navier-Stokes equations. The only difference is that the viscosity now depends on the pressure and strain-rate.

Following Jop et al. (2006), Lagrée et al. (2011) and Staron et al. (2012, 2014), Equation (2) is generalized to complex flow configurations, where stresses may not be spatially uniform, by modeling the viscosity of the granular matter, $\eta_{M C c}$, by

$$
\eta_{M C C}=\max \left(\frac{\mu(I)}{D_{2}} p, 0\right)
$$

where $p$ is the pressure and the inertial number is defined using 
In Equations (7) and (8), $D_{2}=\sqrt{D_{i j} D_{i j}}$ is the second invariant of the strain rate tensor, which is merely a measure of the strain or shear rate that has been generalized to complex three-dimensional flows. In this generalized $\mu(I)$-rheology model it is assumed that the strain rate tensor and the deviatoric part of the overall stress tensor are codirectional.

For the simulations in this work, the viscosity and density of the air phase are assumed to be $1.80 \times 10^{-5} \mathrm{~Pa} \cdot \mathrm{s}$ and $1.22 \mathrm{~kg} / \mathrm{m}^{3}$, respectively. As Equation (7) indicates, the present framework uses a fluid with a very high viscosity to model a solid-like behavior. To avoid numerical instabilities that may arise when the strain rate is small, the viscosity of the granular phase is bounded by $\eta_{M C C, \max }=250 \rho_{0} \sqrt{g H^{3}}$, where $H$ is the initial depth of the powder bed, as suggested by Lagrée et al. (2011). It has been verified that the results presented in this work are not sensitive to this upper bound of the viscosity.

Although it is straightforward to measure the angle of wall friction, we are interested in determining to what extent the wall boundary condition affects the flow behavior. We therefore follow Staron et al. (2012) and carry out simulations using the two limiting wall boundary conditions of no-slip and free-slip. Note that, in both cases, the behavior of the powders is expected to be governed primarily by local conditions created by the outlet of a hopper, since the velocities far from that region will be very small or zero for both types of boundary conditions.

The axisymmetric computational domain is shown in Figure 2 (top). The mass and momentum conservation equations are discretized on a co-located grid using a second-order upwind scheme for the convection terms and a second-order central-differencing scheme for the diffusion terms. The pressure-velocity coupling algorithm is SIMPLE, and the pressure interpolation scheme is PRESTO! (Patankar, 1980). The volume fraction advection equation is discretized using a geometrical reconstruction scheme. The computational mesh, for which the results are presented if not stated otherwise, is shown in Figure 2 (bottom). The simulations are performed using ANSYS ${ }^{\circledR}$ Fluent 17.2 .0 with the $\mu(I)$-rheology model implemented via user-defined functions. 

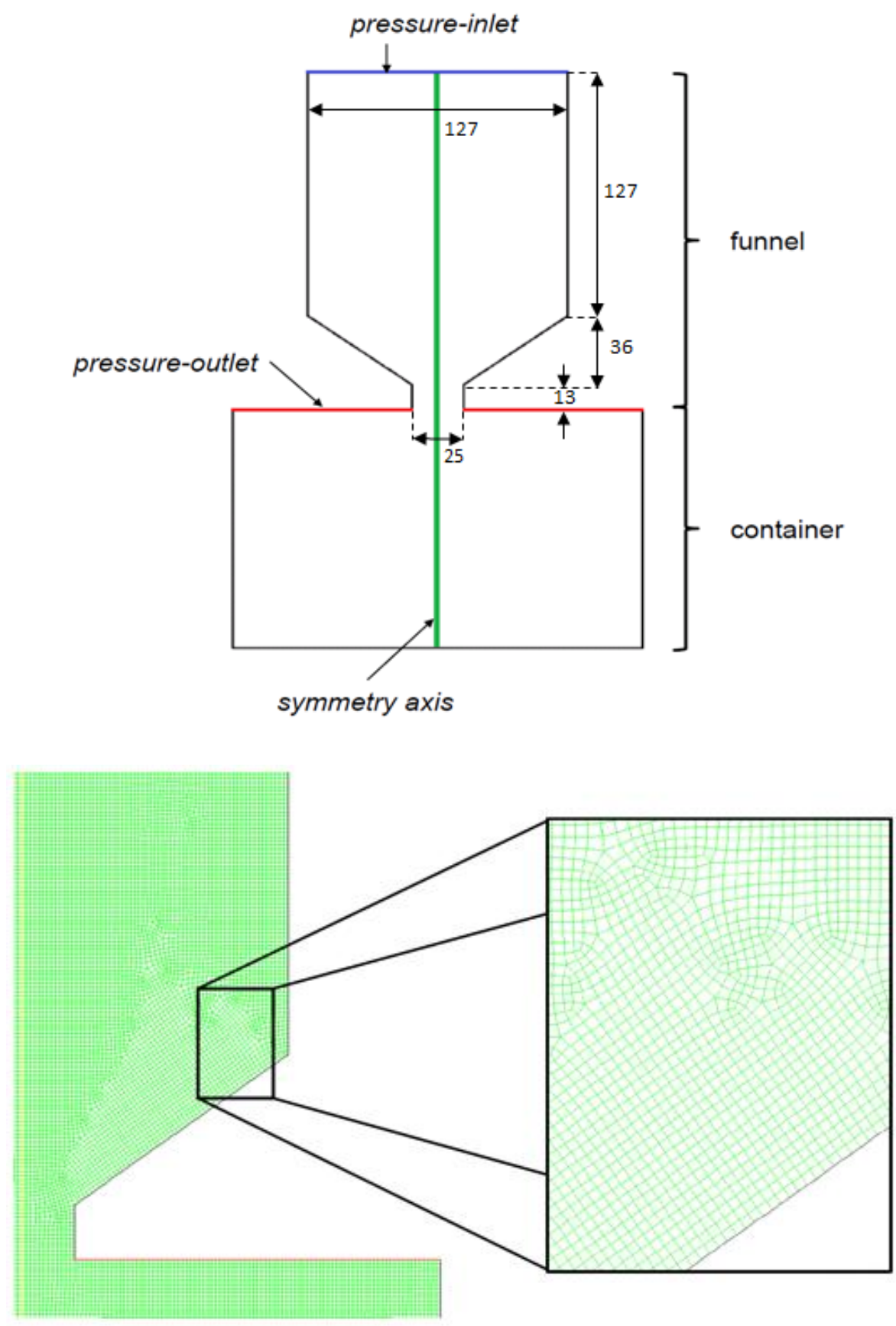

Figure 2: Top: The computational domain. The domain is axisymmetric, but here it is mirrored about the symmetry axis for purposes of illustration. Bottom: The mesh used in the simulations contains 22,023 cells. Most of them are perfect squares but number of non-square hexahedral cells are necessary because of the angles in the funnel. The resolution $\Delta x$ is in the range [2.2, 4.1] $\mathrm{mm}$ and satisfies the requirements of VOF. The figure on the right magnifies the region close to an inclined wall of the hopper. 
The temporal discretization of all equations is first-order implicit, except for the volume fraction advection equation that uses an explicit time stepping. We have seen in our simulations that the shape of the interface changes significantly during the discharge process, and adaptive time stepping controlled via the largest global cell Courant number is therefore employed. As a consequence, the typical time steps are in the interval $10^{-5}-10^{-4} \mathrm{~s}$.

The simulations typically exhibit an induction period before any appreciable flow begins. During this period numerical errors result in a fluctuation in the powder velocity and velocity gradient that causes an increase in the inertial number, a decrease in the effective viscosity of the granular phase, and, consequently, an increase in the flow rate. The duration of this induction period depends on the time stepping and, to a lesser extent, the grid resolution and is thus not physical. After this initial phase, the flow behavior is not sensitive to the numerical details and may thus be compared to the experimental data. Also for these predictions of the discharged mass fraction, a moving average is calculated for a one-to-one comparison to the experimental data.

Finally, it is worth mentioning that the numerical framework used here is similar to the one used to describe flows that are governed by the classical single-phase Navier-Stokes equations. If this framework can be successfully applied also in this case, it obviously has the important implication that there is no need for developing new numerical schemes or undertaking any special measures to carry out the simulations.

\section{Results}

\subsection{Material properties}

The bulk density was determined to be $\rho_{0}=900 \mathrm{~kg} / \mathrm{m}^{3}$. Figure 3 shows a SEM image of a sample of the VIVAPUR MCC Spheres 500. As Figure 3 indicates, the particles are neither perfectly spherical nor monodisperse. Figure 4 shows the particle size distribution. The median particle diameter (D50) was determined to be $d=660 \mu \mathrm{m}$, but the particle size distribution is fairly broad and spans from approximately $500 \mu \mathrm{m}$ to $900 \mu \mathrm{m}$. For this range of particle sizes, the sphericity and aspect ratio were determined to be approximately 0.9 and 0.7 , respectively. Although there is a distribution of particle sizes and shapes, it is 

effects due to the particle size and shape distribution.

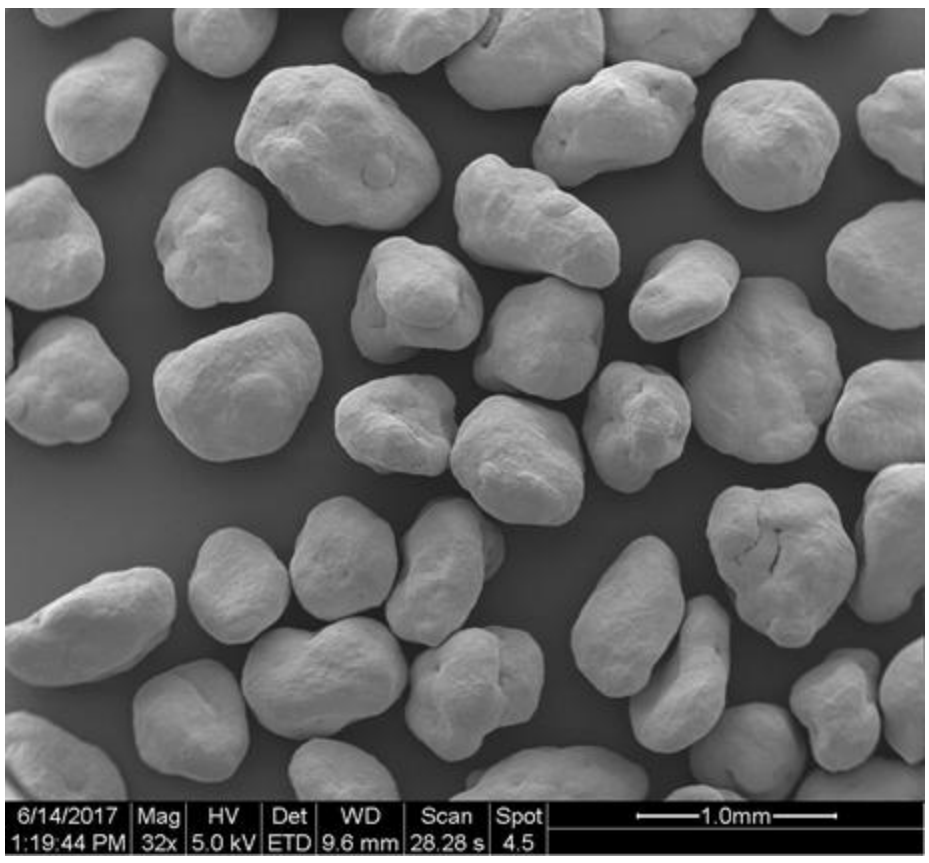

Figure 3: SEM image of the VIVAPUR MCC Spheres 500.

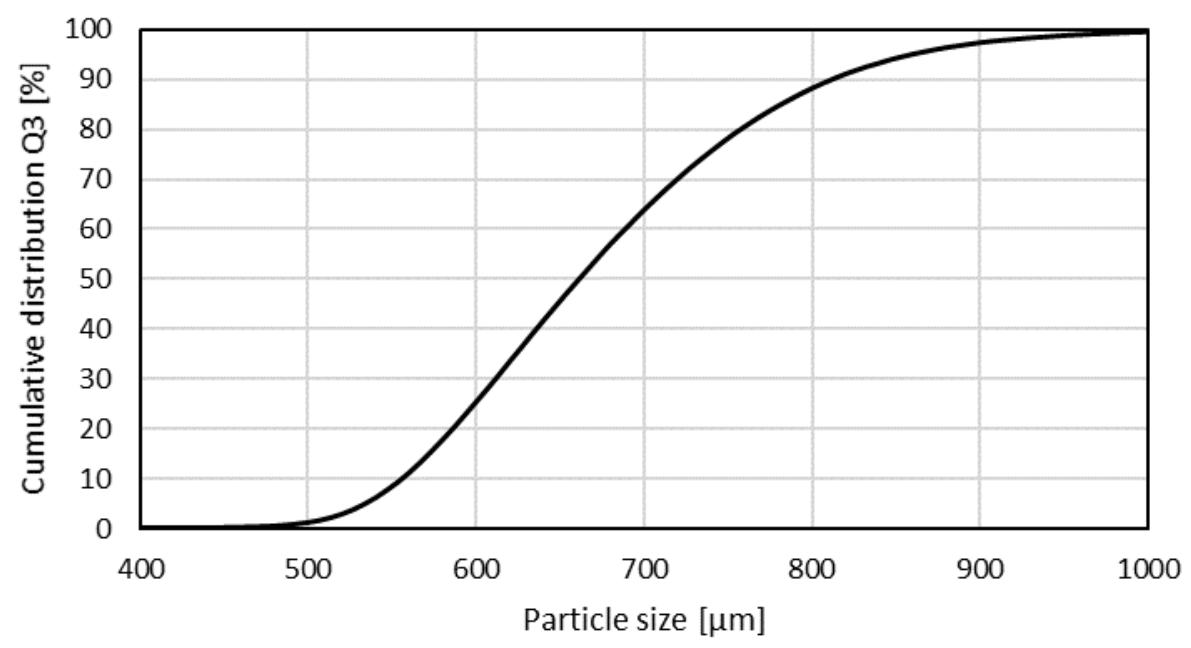

Figure 4. The cumulative particle size distribution of VIVAPUR MCC Spheres 500 used in this work.

328 Using a particle density of $\rho=1500 \mathrm{~kg} / \mathrm{m}^{3}$ (Rowe et al., 2009), the imposed pressure of 400

$329 \mathrm{~Pa}$ and the range of shear rates from 0.002 to $95 \mathrm{~s}^{-1}$ is found to correspond to inertial 
numbers in the range between $10^{-6}$ and 0.1 ; this range covers a wide range of regimes of granular flow, from quasi-static (in which powders have a solid-like behavior) to liquid-like. The latter regime can be characterized by particles moving in such a way that their contacts are not long-lasting, but also that the state of a granular gas is not yet reached (Forterre and Pouliquen, 2008).

Figure 5 shows the effective friction coefficient for the MCC spheres as a function of the inertial number $I$. The friction coefficient tends to a value of 0.401 for low inertial numbers and increases as the inertial number increases. As Figure 5 shows, the experimental data is well described using Equation

(3) with $\mu_{s}=0.401, \mu_{2}=0.908$ and $I_{0}=0.0465$. It would obviously be of interest to characterize the material at higher inertial numbers than approximately 0.1. However, as we will see, such a characterization is not critical for the prediction of the present discharge experiments.

Using the dimensions of the hopper and the bulk density of the powder, the total depth of the powder bed can be estimated to be $H=109 \mathrm{~mm}$, which implies that the transition between the upper cylindrical part of the hopper and the converging section is $72 \mathrm{~mm}$ below the surface of the powder bed. At this transition, the pressure in the upper cylindrical part of the hopper obtained using Equation (5) is between $400 \mathrm{~Pa}$ and $600 \mathrm{~Pa}$ for $\mu_{W}<1$. The experimental characterization of the rheology of the material was thus carried out at a relevant pressure. 


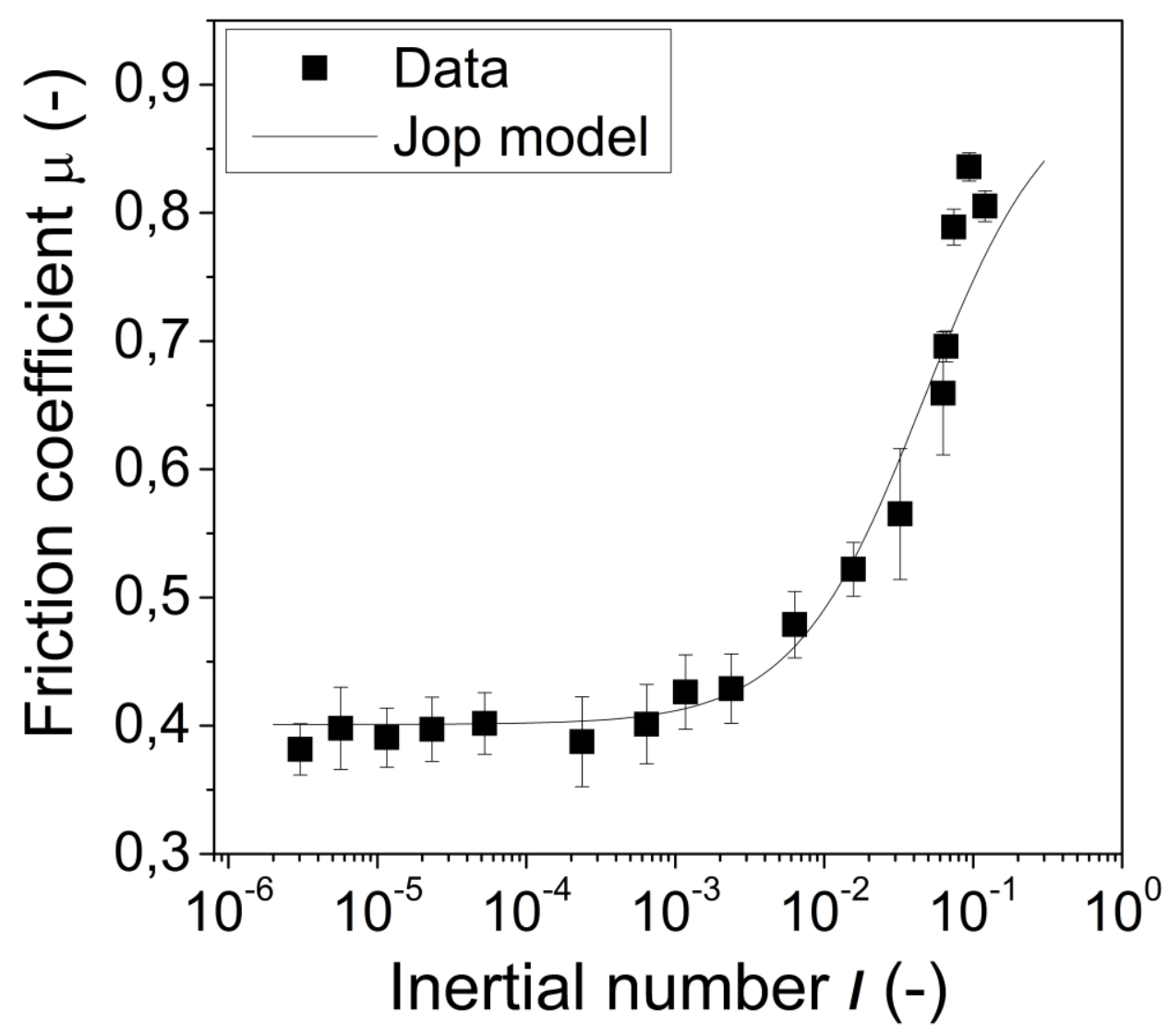

351

352

353

354

355

356

357

358

359

360

361

362

363

Figure 5: The friction coefficient as a function of the inertial number for VIVAPUR MCC Spheres 500.

The solid line represents our fit of the data to the model of Jop et al. (2006).

Figure 6 shows the solids volume fraction as a function of the inertial number, i.e. the dynamic dilatancy law. The latter law implies that an increase in the flow rate leads to a decrease in the packing fraction of the powder: the material dilates under flow. The data can be described using Equation (5) with with $\phi_{\max }=0.632$ and $\phi_{\min }=0.443$.

The increase of the friction coefficient and the decrease of the solids volume fraction for moderate values of the inertial number show the usual behavior of granular materials under shear flow and is similar to other experimental and numerical studies on dry (Fall et al., 2015; GDR Midi, 2004; Hatano 2007; Jop et al., 2006) and wet grains (Berger et al., 2015; Khamseh et al., 2015). The results in Figures 5 and 6 can be combined to show a linear decrease in the effective friction coefficient with an increase in the solid volume fraction, 
which is a very strong result for dry, partially wet, or fully wet granular materials regardless

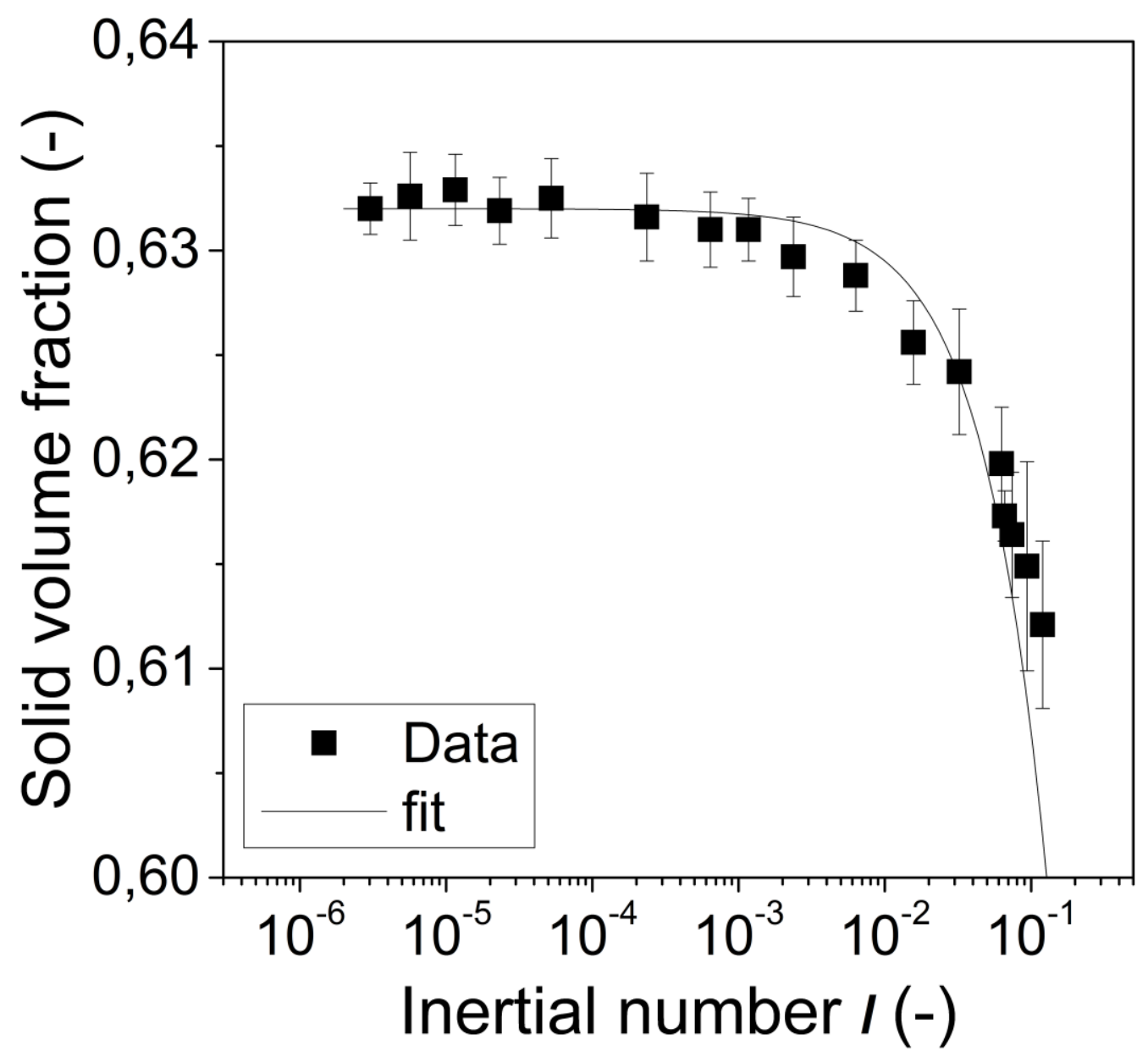

Figure 6: Dynamic dilatancy law - the solid volume fraction as a function of the inertial number for VIVAPUR MCC Spheres 500. The solid line represents the fit of the data to the model of da Cruz et al. (2005).

\subsection{Hopper discharge}

373 Figure 7 shows the simulated and the measured discharge mass fractions as functions of 374 time. Figure 7 also shows the discharge rate that is predicted using the Beverloo correlation 375 in Equation (6) using $k=1$, which will be discussed in the next section. The simulated 
discharge process agrees qualitatively with the measured one in that both the predictions and measurement show a constant discharge rate for the major portion of the discharge process. There is a very small difference between the results obtained for the two boundary conditions, which is in agreement with the observation made by Staron et al. (2012). Also, for both boundary conditions the hopper is emptied completely.

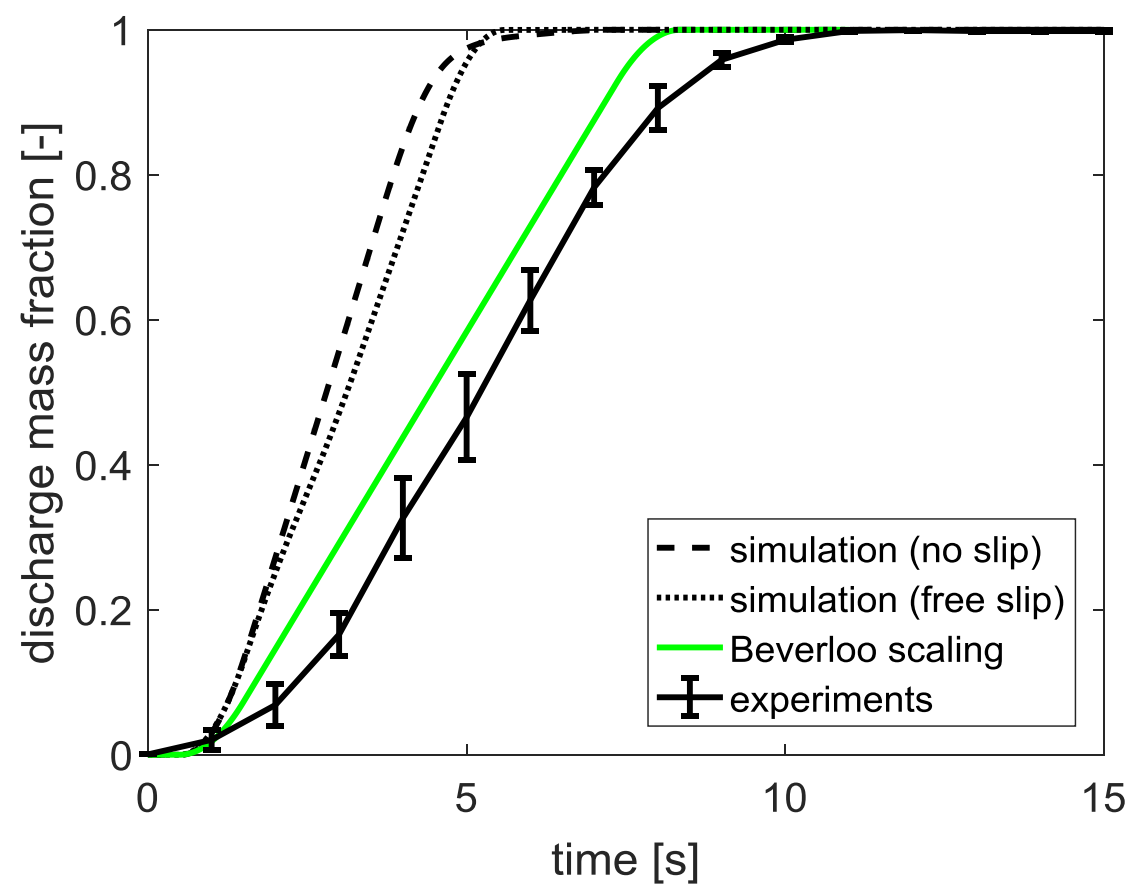

Figure 7: The discharge of MCC spheres from the hopper as a function of time. The error bars represent the experimental uncertainty as quantified by one standard deviation based upon three replicates. Note the correct prediction of the discharge rate obtained using the Beverloo correlation with $k=1$.

Figure 8 shows the simulated volume fraction distribution at three instants in time during discharge of the MCC spheres from the hopper. For the no-slip wall boundary condition, the figure reveals that the shape of the free surface in the hopper is in reasonable agreement with experimental observations in that there is a small but noticeable characteristic dip (sink) in the surface. Interestingly, that sink is not present or barely present for the case of the free-slip wall boundary condition. This difference in the shape of the surface for the two boundary conditions is in contrast to the results obtained by Staron et al. (2012), who found 
that the shape of the surface differed mainly near the wall. The difference is not surprising, however, since Staron and coworkers studied a two-dimensional silo with a ratio of the upper width to the orifice width of approximately 6 , whereas we study an axisymmetric hopper for which the ratio between the diameter of the upper cylinder and the outlet is approximately 10. In addition, Staron et al. (2012) studied a different material. The material behaves slightly differently between the two boundary conditions at the bottom of the container too, but a heap of material is formed at the bottom of the container in both cases. In this context we note that numerical artefacts inherent to the implemented interfacecapturing method affect the shape of the pile below the hopper. We have not focused on resolving these issues since these numerical artefacts affect neither the flow pattern in the hopper nor the discharge rate.

$$
t=0.5 \mathrm{~s}
$$

$$
t=2 \mathrm{~s}
$$$$
t=4 \mathrm{~s}
$$

no slip
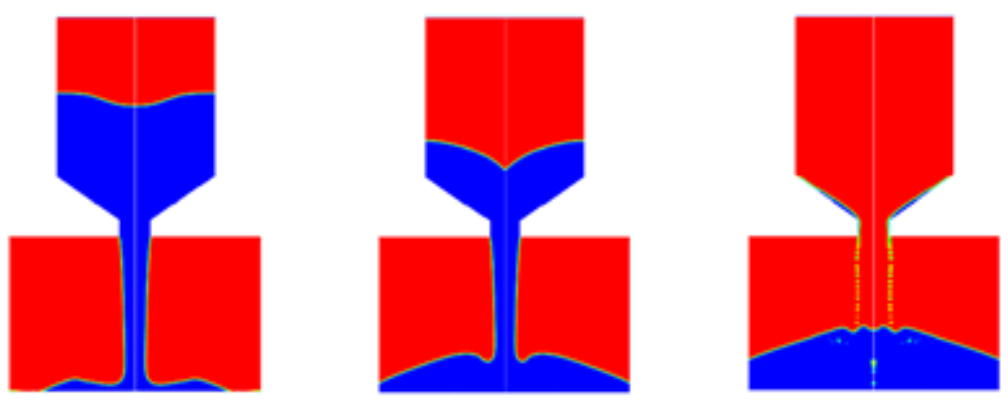

\section{free slip}
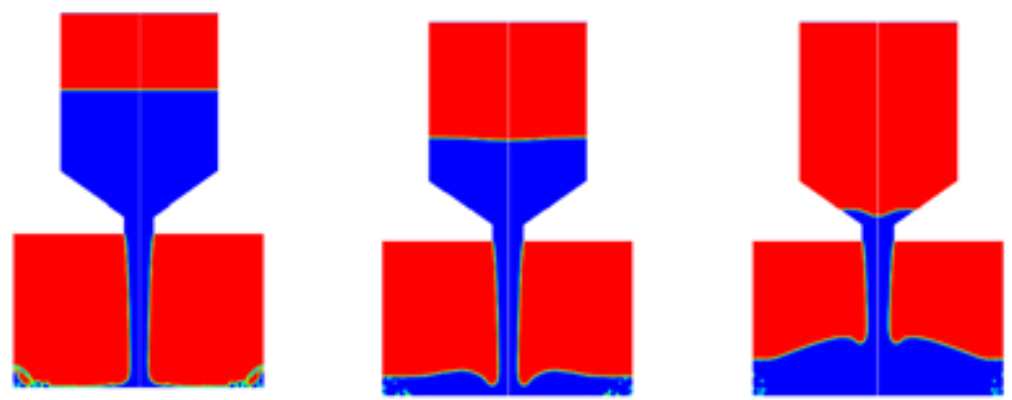

Figure 8: The simulated discharge of MCC 500 spheres from the hopper as a function of time (top: noslip boundary condition at the walls, bottom: free-slip boundary condition at the walls). Blue: MCC particles, red: air. The droplet-like features on the bottom corners of the container for the free-slip boundary condition at $t=0.5 \mathrm{~s}$ are numerical artefacts that typically are obtained when using a VOF method. 
The fact that the no-slip boundary condition results in a higher discharge rate is explained by noting that different velocity fields induce different viscosity fields. In the simulation that employs a free-slip boundary condition, the top section of the granular bed moves as a solidlike region for the first one or two seconds, as evidenced by the almost flat gas-solid interface at the top of the powder for this boundary condition (see Figure 8). The difference in the velocity fields also reveal in important difference between the two boundary conditions: as indicated in Figure 9, the free-slip boundary condition predicts a velocity field that is reminiscent of a so-called radial velocity field that is associated with mass flow bins (Jenike, 1961), whereas the no slip boundary condition predicts a velocity field that more closely resembles funnel flow. In this regard, the results obtained using the no-slip boundary condition are in better agreement with the experimental data. In connection with Figure 9, it is interesting to note that the discharge rate is so similar for the two boundary conditions despite the large difference between the velocity fields.

$$
t=0.5 \mathrm{~s} \quad t=1 \mathrm{~s} \quad t=2 \mathrm{~s} \quad t=3 \mathrm{~s} \quad t=4 \mathrm{~s}
$$
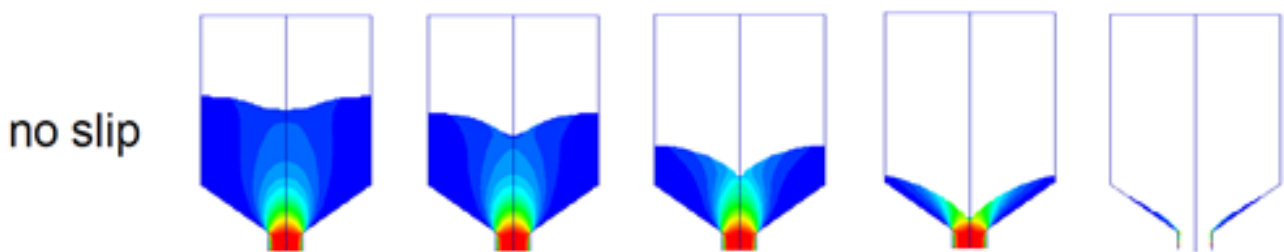

0.75
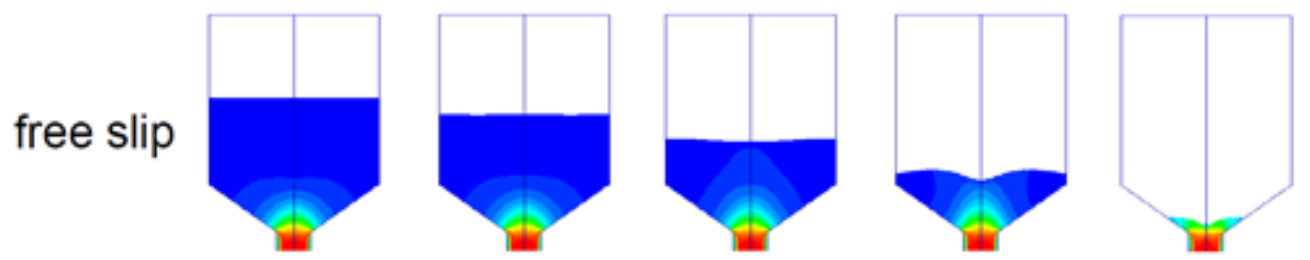

Figure 9: The velocity magnitude in the MCC powder at different points in time during the discharge process for the no-slip and free-slip wall boundary conditions. For the purpose of visualization, the color scale is saturated at $0.75 \mathrm{~m} / \mathrm{s}$, meaning that the actual velocities in the funnel outlet may be slightly higher. The simulations are carried out with a mesh resolution shown in Figure 2 (bottom).

In order to understand the different regimes of granular flow present in various regions of the hopper, the predicted viscosity (Equation (7)) at different points in time during the 
discharge process is shown in Figure 10 (for the no-slip boundary condition). It is straightforward to identify three regions of flow in the hopper with markedly different viscosities: a low-viscosity region close to the outlet where the granular material behaves like a gas, a medium-viscosity region above the outlet where the material behaves like a liquid, and a high-viscosity region close to the inclined walls where the granular material behaves more like a solid. This behavior obviously results in steep velocity gradients in the hopper, as seen in Figure 9.

$$
t=0.5 \mathrm{~s}
$$

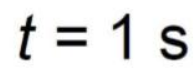

$$
t=2 \mathrm{~s}
$$$$
t=3 \mathrm{~s}
$$

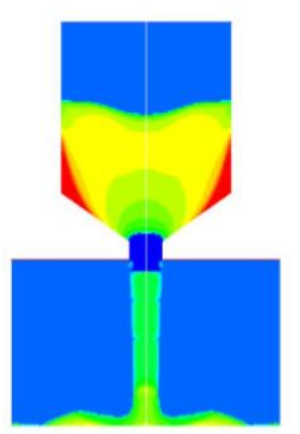

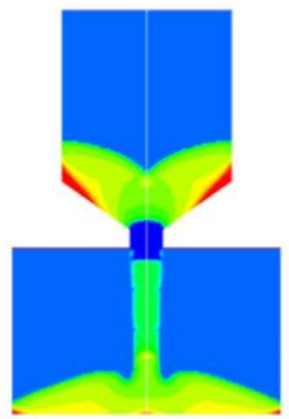

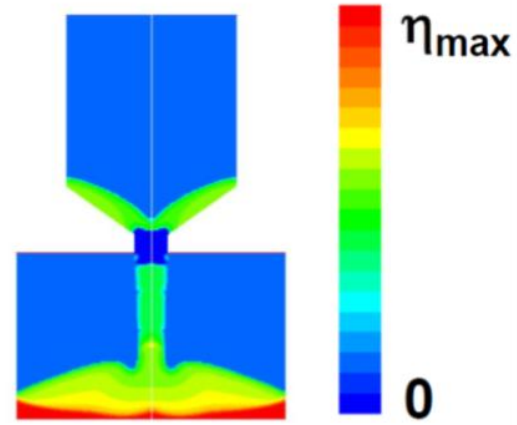

Figure 10: The viscosity at different points in time during the discharge process of MCC powders for the no-slip wall boundary condition. The color scale is logarithmic, with an upper-bound value of $\eta_{M C C, \max }=250 \rho_{0} \sqrt{g H^{3}}$ (Lagree et al., 2011). The simulations are carried out with a mesh resolution shown in Figure 2 (bottom).

Lastly, Figure 11 shows the inertial number in the granular phase at different points in time during the discharge process. There is a wide range in the inertial number, which varies from approximately $10^{-8}$ to $10^{2}$. In this context, we note that the effective friction coefficient has a low inertial number asymptote for $I<10^{-3}$ and a high inertial number asymptote for $I>1$. Figure 11 shows that the regions where the inertial number is high $(I>1)$ correspond to regions where the material has a low viscosity. Conversely, the regions where the inertial number is low $\left(I<10^{-3}\right)$ correspond to regions where the viscosity is high. As already noted, the inertial number just above the outlet is approximately 0.5 , which is slightly higher than the maximum inertial number for which it was possible to characterize the material using the technique described in this work. Developing a technique that allows for 
463

464

characterization at higher inertial numbers is obviously of general interest, and in this case it would improve the reliability of the parameter $\mu_{2}$. However, in the case of discharge of the present MCC spheres from this specific hopper, we have carried out simulations that show that the discharge rate is not particularly sensitive to the value of this parameter.
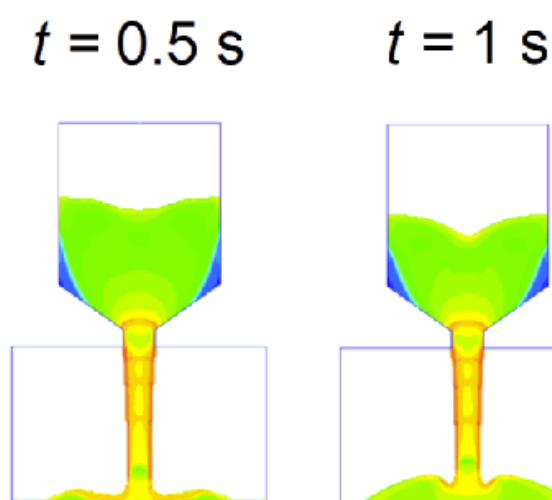

$$
t=2 \mathrm{~s}
$$
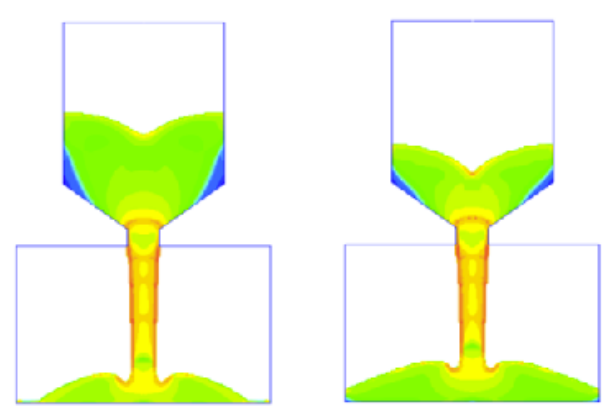
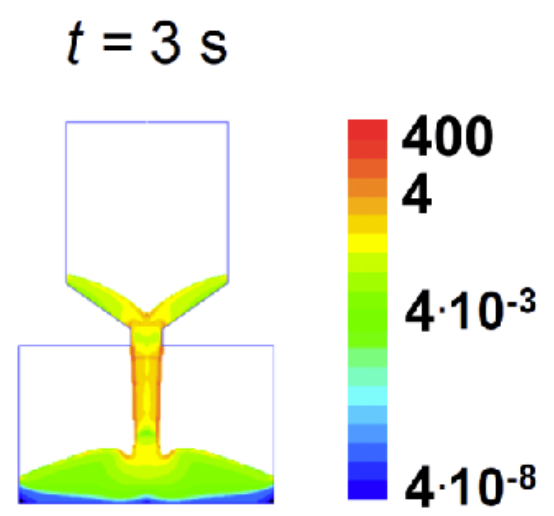

Figure 11: The predicted inertial number at several instants in time during the discharge process of MCC powders for the no-slip wall boundary condition. The values of the inertial number identify different regimes of granular flow in the hopper (solid-like, liquid-like and gas-like). The simulations are carried out with a mesh resolution shown in Figure 2 (bottom).

\section{Discussion}

While there is qualitative agreement between the predicted and measured discharge processes, the predicted discharge rate does not agree quantitatively with the measured discharge rate. To facilitate a comparison between the discharge rates, the mean discharge rates during the steady phase have been extracted and are presented in Table 1 along with the discharge rate predicted using the Beverloo correlation in Equation (6). The discharge rate predicted by the Beverloo correlation depends slightly on the value of $k$, but for $1<k<$ 3 , Table 1 shows, that the discharge rate is predicted better using the Beverloo correlation than the discharge simulation based on the $\mu(I)$-rheology model.

Table 1. The measured and predicted discharge rate during the steady phase. 


\begin{tabular}{|l|l|}
\hline Case & Discharge rate during the steady phase $[\mathrm{g} / \mathrm{s}]$ \\
\hline Experiment & 161 \\
\hline Beverloo, $k=1$ & 151 \\
\hline Beverloo, $k=3$ & 131 \\
\hline Simulation, free-slip & 253 \\
\hline Simulation, no-slip & 302 \\
\hline Simulation, no-slip refined & 222 \\
\hline
\end{tabular}

In the literature, the Beverloo scaling is sometimes attributed to the so-called Janssen effect (Ovarlez et al., 2003 and Sperl, 2006), i.e. that friction at the wall prevents the lower region of the hopper or silo from sensing the weight of the material. While this certainly is true in the static case, the results presented here show that the Janssen effect may not be responsible for the Beverloo scaling. The discharge rate is rather controlled by the mechanism that drives the grains out of the container: the acceleration due to the gravity. In particular, Figure 7 shows that a constant discharge rate is obtained even when there is no friction at the wall, i.e. for the free-slip boundary condition. The results presented here are thus consistent with the conclusion of Staron et al. (2012), who suggested that the Beverloo scaling results from the yield stress properties of the material.

We will now turn to a discussion on the general suitability of the present framework to simulate the discharge from a hopper and on how the agreement between the experiments and predictions can be improved. Our work shows that in the regions where the material is predicted to have a very low viscosity, i.e. the regions where the inertial number is very high, the model is not expected to be valid since the material behaves like a granular gas. In the present case and most likely many other cases, this may not be much of an issue since the flow pattern in any case is dominated by the behavior in regions where the viscosity is high. More importantly, however, the present model is also not expected to be valid in the regions where the material is predicted to have a very high viscosity, i.e. in the regions where the material behavior is solid-like. In the present implementation of the model, this behavior has been approximated using a very high viscosity; the results indicate that a reasonable prediction of the discharge behavior in this case is obtained despite this simplifying assumption. In other cases, however, a more accurate description of the material behavior 
in these regions may be required. As noted by Jop et al. (2006), developing a predictive capability for the transition between the solid-like and liquid-like behavior at low inertial numbers can be challenging and it is likely that information on the level of a single particle becomes important. Although it is an open question how to include these solid-like regions and their possible behaviors in a continuum description of powder flow, the recent work of Kamrin and Koval (2012) and Henann and Kamrin (2013) to develop a grain-size-dependent model is very encouraging.

A straightforward step in obtaining better agreement between the simulated and experimental results is to refine the computational mesh. This type of action exposes one important feature of the numerical framework: while it is possible to obtain a solution that shows better agreement with the measurements than the one shown in Figure 7, we have not been able to perform simulations using increasingly fine computational grids to obtain a grid-independent discharge rate. In other words, we have indeed found that the experimental discharge rate can be better reproduced when employing a finer grid resolution. One such example is shown in Figure 12, in which the mesh resolution is twice as fine as the one shown in Figure 2 (bottom). However, the simulations produce physically unrealistic predictions as the grid resolution increases beyond some critical point. These observations agree with the analysis of Barker et al. (2015), who showed that the model is ill-posed. That is, as the grid is refined, short-wavelength disturbances grow without bound and render the solution physically meaningless, in contrast to convergence with respect to increasing mesh resolution that is expected for models that are mathematically sound. To address this issue, Barker and Gray (2017) proposed a regularized version of the $\mu(I)$ rheology model. Although an evaluation of the regularized model is very interesting, it is beyond the scope of the present study. In a forthcoming work, we aim to perform a more comprehensive investigation into the model behavior at very fine mesh resolution in order to explore this issue further. 


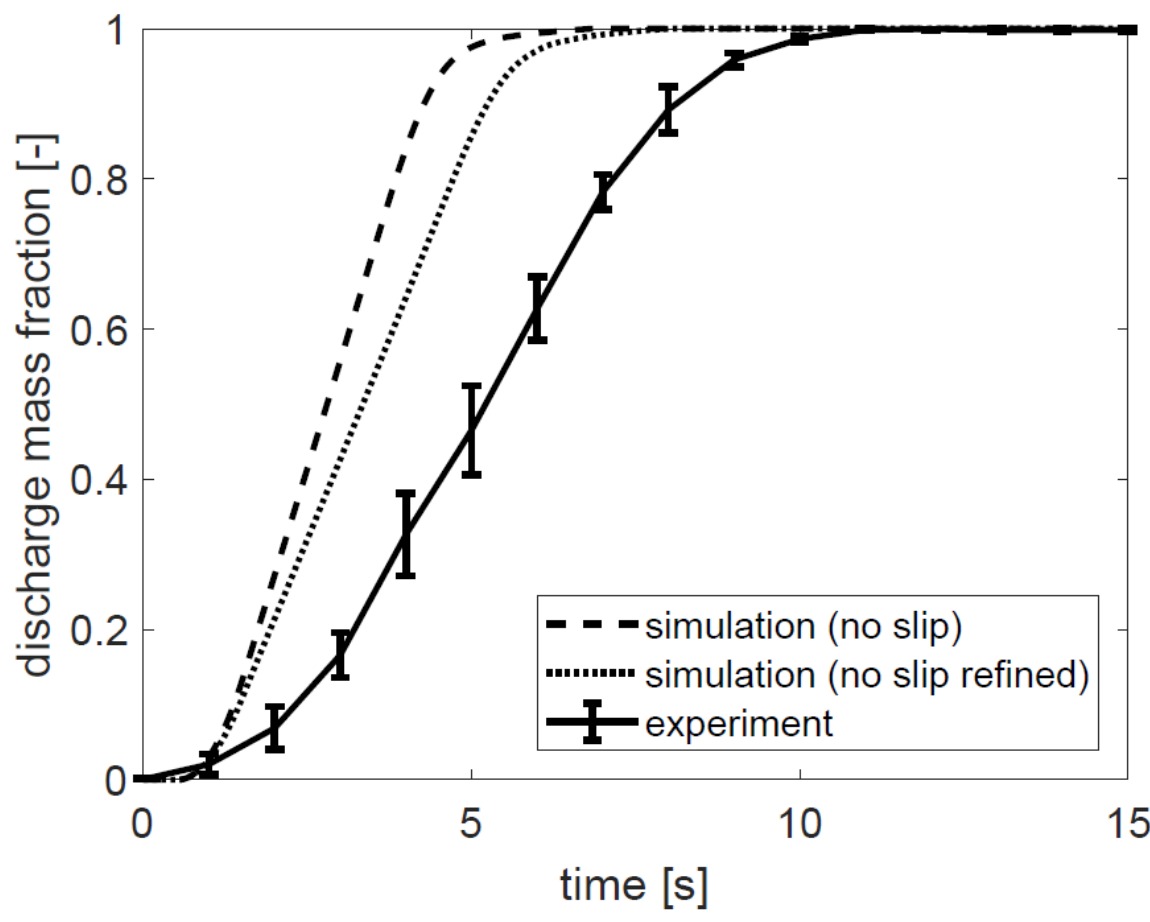

530

Figure 12: The simulated discharge of MCC spheres from the hopper as a function of time (no-slip boundary condition at the walls). The refined mesh-case uses a resolution that is twice as fine as that shown in Figure 2 (bottom). The error bars represent the experimental uncertainty as quantified by one standard deviation based upon three replicates.

In addition to problems related to the ill-posedness of the current $\mu(I)$-model in describing the rheology of the granular material, there are additional limitations in the present implementation of the model. For example, the VOF method does not consider changes in the solids volume volume fraction, which have an effect on the effective friction coefficient. In addition, the VOF method does not take into account the flow of the continuous fluid through the powder and the corresponding momentum transfer between the phases (i.e. the interphase drag). To establish a predictive capability that is generally applicable, it is necessary to include this interaction. Including a granular phase in which the volume fraction of the powder is allowed to vary and that includes interphase drag may not be very important for the flow of the present MCC spheres from an open-ended hopper but may be more important in other situations. For example, for powder flow from an intermediate bulk container in a fully contained pharmaceutical manufacturing process there is a backflow of air that can affect the discharge rate and lead to segregation. Including these effects is 
therefore particularly relevant for many pharmaceutical materials, which often have a particle size that is on the order of $10-100 \mu \mathrm{m}$ and for which aerodynamic drag can be expected to be important.

\section{Conclusions}

This work shows that the rheology of a typical pharmaceutical material can be measured using a slightly modified annular shear rheometer and that the results can be interpreted in terms of the $\mu(I)$-rheology model. It is demonstrated that this model, implemented in a VOF framework, is capable of qualitatively predicting the discharge of VIVAPUR MCC Spheres 500 from a small-scale hopper.

While the experimental data as well as the model prediction show constant discharge rates, there is a discrepancy in the value of the discharge rate. In an attempt to find a gridindependent discharge rate for a quantitative comparison with experimental data, it is found that the predicted discharge process becomes physically unrealistic as the grid resolution is increased beyond some critical point. These observations agree with the analysis due to Barker et al. (2015), who found that the model is inherently ill-posed.

Lastly, it is argued that a generally applicable implementation of the model must include a powder phase in which the powder volume fraction can vary as well as interphase drag and the effect of the interstitial air. While interphase drag may not be very important for the flow of the present MCC spheres, it is expected to be important for a majority of pharmaceutical powders.

\section{Acknowledgements}

This research did not receive any specific grant from funding agencies in the public, commercial, or not-for-profit sectors.

\section{References}


Anand, A., Curtis, J.S., Wassgren, C.R., Hancock, B.C., Ketterhagen, W.R., 2008. Predicting discharge dynamics from a rectangular hopper using the discrete element method (DEM). Chem. Eng. Sci. 63, 5821-5830.

Anand, A., Curtis, J.S., Wassgren, C.R., Hancock, B.C., Ketterhagen, W.R., 2009. Predicting discharge dynamics of wet cohesive particles from a rectangular hopper using the discrete element method (DEM). Chem. Eng. Sci. 64, 5268-5275.

Ancey, C., Coussot, P., Evesque, P., 1999. A theoretical framework for granular suspensions in a steady simple shear flow. J. Rheol. 43, 1673-1699.

Andreotti, B.Y., Forterre, Y., Pouliquen, O., 2013. Granular Media: Between Fluid and Solid. Cambruidge University Press, Cambridge, UK.

ASTM D6940-03, 2003. Standard Practice for Measuring Sifting Segregation Tendencies of Bulk Solids. ASTM International, West Conshohocken, PA.

Bai, L., Zheng, Q.J., Yu, A.B., 2017. FEM simulation of particle flow and convective mixing in a cylindrical bladed mixer. Powder Technol. 313, 175-183.

Barker, T., Schaeffer, D. G., Bohorquez, P., Gray, J. M. N. T., 2015. Well-posed and ill-posed behavior of the $\mu(I)$-rheology for granular flow. J. Fluid Mech. 779, 794-818.

Barker, T., Gray, J.M.N.T., 2017. Partial regularization of the incompressible $\mu(I)-$-rheology for granular flow. J. Fluid Mech. 828, 5-32.

Berger, N., Azéma, E., Douce, J.-F., Radjai, F., 2015. Scaling behaviour of cohesive granular flows. Europhys. Lett. 112, 64004.

Beverloo, W.A., Leninger, H.A., van de Valde, J., 1961. The flow of granular solids through orifices. Chem. Eng. Sci. 15, 260.

Bierwish, C., Kraft, T., Riedel, H., Moseler, M, 2009. Three-dimensional discrete element models for the granular statics and dynamics of powders in cavity filling. J. Mech. Phys. Solids 57, 10-31.

Campbell, C.S., 1990. Rapid granular flows. Annu. Rev. Fluid Mech. 22, 57-92.

Campbell, C.S., 2006. Granular material flows - An overview. Powder Technol. 162, 208-229.

Chen, X., Wang, J., 2014. A comparison of two-fluid model, dense discrete particle model and CFDDEM method for modeling impinging gas-solid flows. Powder Technol. 254, 94-102. 
604

605

606

607

608

609

610

611

612

613

614

615

616

617

618

619

620

621

622

623

624

625

626

627

628

629

630

631

Chialvo, S., Sun, J., Sundaresan, S., 2012. Bridging the rheology of granular flows in three regimes. Phys. Rev. E 85, 021305.

Cox, E.P., 1927. A method of assigning numerical and percentage values to the degree of roundness of sand grains. J. Paleontol. 1, 179-183.

da Cruz, F., Emam, S., Prochnow, M., Roux, J.-N., Chevoir, F., 2005. Rheophysics of dense granular materials: Discrete simulation of plane shear flows. Phys. Rev. E 72, 021309.

Dunatunga, S., Kamrin, K., 2015. Continuum modeling and simulation of granular flows through their many phases. J. Fluid Mech. 779, 483-513.

European Pharmacopoeia $9^{\text {th }}$ Edition (9.3), 2018. 2.9.34 Bulk density and tapped density of powders.

Fall A., Ovarlez G., Hautemayou D., Mézière C., Roux, J.-N., Chevoir, F., 2015. Dry granular flows:

rheological measurements of the $\mu(I)$-rheology. J. Rheol. 59, 1065-1080.

Farzaneh, M., Almstedt, A.E., Johnsson, F., Pallarès, D., Sasic, S., 2015. The crucial role of frictional stress models for simulation of bubbling fluidized beds. Powder Technol. 270, 68-82.

Forterre, Y. and Pouliquen, O., 2008. Flows of dense granular media. Annu. Rev. Fluid Mech. 40, 1-24.

GDR Midi, 2004. On dense granular flows. Eur. Phys. J. E 14, 341-65.

Hatano, T., 2007. Power-law friction in closely packed granular materials. Phys. Rev. E 75, 060301(R).

Henann, D.L., Kamrin, K., 2013. A predictive, size-dependent continuum model for dense granular flows. P. Natl. Acad. Sci. USA 110, 6730-6735.

Janssen, H.A., 1895. Getreidedruck in Silozellen. Z. VDI 30, 1045-1049.

Jenike, A.W., 1961. Gravity flow of bulk solids. Bulletin 108, Utah Engineering Experiment Station, University of Utah, 1961.

Jenike, A.W., Johanson, J.R., Carson, J.W., 1973. Bin loads - part 3: Mass flow bins. Trans. ASME, Ser. B 95, 6-12.

Jop, P., Forterre, Y., Pouliquen, O., 2006. A constitutive relation for dense granular flow. Nature 441, 727-730.

Kamrin, K., Koval, G., 2012. Nonlocal constitutive relation for steady granular flow. Phys. Rev. Lett. $108,178301$. 
632

633

634

635

636

637

638

639

640

641

642

643

644

645

646

647

648

649

650

651

652

653

654

655

656

657

658

659

660

Ketterhagen, W.R., Curtis, J.S., Wassgren, C.R., Hancock, B.C., 2009. Predicting the flow mode from hoppers using the discrete element method. Powder Technol. 195, 1-10.

Khamseh, S., Roux, J.-N., Chevoir, F., 2015. Flow of wet granular materials: A numerical study. Phys. Rev. E 92, 22201.

Lagrée, P.Y., Staron, L., Popinet, S., 2011. The granular column as a continuum: validity of a twodimensional Navier-Stokes model with a $\mu$-rheology. J. Fluid Mech. 686, 378-408.

Liu, Y., Cameron, A.T., Gonzalez, M., Wassgren, C., 2018a. Modeling granular material blending in a Tote blender using a finite element method and advection-diffusion equation multi-scale model. Powder Technol. 340, 428-439.

Liu, Y., Gonzalez, M., Wassgren, C., 2018b. Modeling granular material blending in a rotating drum using a finite element method and advection-diffusion equation multiscale model. AIChE J. 64, 32773292.

Nedderman, R., Tuzun, U., Savage, S., Houlsby, G., 1982. The flow of granular-materials .1. Discharge rates from hoppers. Chem. Eng. Sci. 37, 1597-1609.

Ovarlez, G., Fond, C., Clement, E., 2003. Overshoot effect in the Janssen granular column: A crucial test for granular mechanics. Phys. Rev. E 67, 060302.

Patankar, S.V., 1980. Numerical Heat Transfer and Fluid Flow. Hemisphere, Washington, DC.

Persson, A.-S., Alderborn, G., Frenning, G., 2011. Flowability of surface modified pharmaceutical granules: A comparative experimental and numerical study. Eur. J. Pharm. Sci. 42, 199-209.

Popoff, B., Braun, M., 2007. A Lagrange approach to dense particulate flows. International Conference on Multiphase Flows, Leipzig, Germany.

Queteschiner, D., Lichtenegger, T., Pirker, S., Schneiderbauer, S., 2018. Multi-level coarse-grain model of the DEM. Powder Technol. 338, 614-624.

Rognon, P.G., Roux, J.-N., Naaïm, M., Chevoir, F., 2008. Dense flows of cohesive granular materials. J. Fluid Mech. 596, 21-47.

Rowe, R.C., Sheskey, P.J., Quinn, M.E. (eds.), 2009. Handbook of Pharmaceutical Excipients, $6^{\text {th }}$ edition. Pharmaceutical Press, London.

Savage, S.B., Hutter, K., 1989. The motion of a finite mass of granular material down a rough incline.

J. Fluid Mech. 199, 177-215. 
661

662

663

664

665

666

667

668

669

670

671

672

673

674

675

676

677

678

Schneiderbauer, S., Aigner, A., Pirker, S., 2012. A comprehensive frictional-kinetic model for gasparticle flows: Analysis of fluidized and moving bed regimes. Chem. Eng. Sci. 80, 279-292.

Sperl, M., 2006. Experiments on corn pressure in silo cells translation and comment on Janssen's paper from 1895. Granul. Matter 8, 59-65.

Spink, C.D., Nedderman, R.M., 1978. Gravity discharge rate of fine particles from hoppers. Powder Technol. 21, 245-261.

Staron, L., Lagrée, P.Y., Popinet, S., 2012. The granular silo as a continuum plastic flow: the hourglass and the clepsydra. Phys. Fluids 24, 103301.

Staron, L., Lagrée, P.Y., Popinet, S., 2014. Continuum simulation of the discharge of the granular silo.Eur. Phys. J. E 37, 5.

Zheng, Q.J., Yu, A.B., 2015a. Finite element investigation of the flow and stress patterns in conical hopper during discharge. Chem. Eng. Sci. 129, 49-57.

Zheng, Q.J., Yu, A.B., 2015b. Modelling the granular flow in a rotating drum by the Eulerian finite element method. Powder Technol. 286, 361-370.

Zheng, Q.J., Xia, B.S., Pan, R.H., Yu, A.B., 2017a. Piping flow of cohesive granular materials in silo modelled by finite element method. Granul. Matter 19, 2.

Zheng, Q.J., Xia, B.S., Pan, R.H., Yu, A.B., 2017b. Prediction of mass discharge rate in conical hoppers using elastoplastic model. Powder Technol. 307, 63-72. 


\section{Figure captions}

Figure 1: Sketch of a modified Malvern Kinexus rheometer used in this work for characterizing pharmaceutical powders (Fall et al., 2015).

Figure 2: Top: The computational domain. The domain is axisymmetric, but here it is mirrored about the symmetry axis for purposes of illustration. Bottom: The mesh used in the simulations contains 22,023 cells. Most of them are perfect squares but number of non-square hexahedral cells are necessary because of the angles in the funnel. The resolution $\Delta x$ is in the range $[2.2,4.1] \mathrm{mm}$ and satisfies the requirements of VOF. The figure on the right magnifies the region close to an inclined wall of the hopper.

Figure 3: SEM image of the VIVAPUR MCC Spheres 500.

Figure 4. The cumulative particle size distribution of VIVAPUR MCC Spheres 500 used in this work.

Figure 5: The friction coefficient as a function of the inertial number for VIVAPUR MCC Spheres 500. The solid line represents our fit of the data to the model of Jop et al. (2006).

Figure 6: Dynamic dilatancy law - the solid volume fraction as a function of the inertial number for VIVAPUR MCC Spheres 500. The solid line represents the fit of the data to the model of da Cruz et al. (2005).

Figure 7: The discharge of MCC spheres from the hopper as a function of time. The error bars represent the experimental uncertainty as quantified by one standard deviation based upon three replicates. Note the correct prediction of the discharge rate obtained using the Beverloo correlation with $\mathrm{k}=1$.

Figure 8: The simulated discharge of MCC 500 spheres from the hopper as a function of time (top: no-slip boundary condition at the walls, bottom: free-slip boundary condition at the walls). Blue: MCC particles, red: air. The droplet-like features on the bottom corners of the container for the freeslip boundary condition at $\mathrm{t}=0.5 \mathrm{~s}$ are numerical artefacts that typically are obtained when $u$ sing a VOF method.

Figure 9: The velocity magnitude in the MCC powder at different points in time during the discharge process for the no-slip and free-slip wall boundary conditions. For the purpose of visualization, the color scale is saturated at $0.75 \mathrm{~m} / \mathrm{s}$, meaning that the actual velocities in the funnel outlet may be slightly higher. The simulations are carried out with a mesh resolution shown in Figure 2 (bottom). 
Figure 10: The viscosity at different points in time during the discharge process of MCC powders for the no-slip wall boundary condition. The color scale is logarithmic, with an upper-bound value of $\eta_{M C C, \max }=250 \rho_{0} \sqrt{g H^{3}}$ (Lagree et al., 2011). The simulations are carried out with a mesh resolution shown in Figure 2 (bottom).

Figure 11: The predicted inertial number at several instants in time during the discharge process of MCC powders for the no-slip wall boundary condition. The values of the inertial number identify different regimes of granular flow in the hopper (solid-like, liquid-like and gas-like). The simulations are carried out with a mesh resolution shown in Figure 2 (bottom).

Figure 12: The simulated discharge of MCC spheres from the hopper as a function of time (no-slip boundary condition at the walls). The refined mesh-case uses a resolution that is twice as fine as that shown in Figure 2 (bottom). The error bars represent the experimental uncertainty as quantified by one standard deviation based upon three replicates. 
Click here to download high resolution image

\section{RHEOMETER}
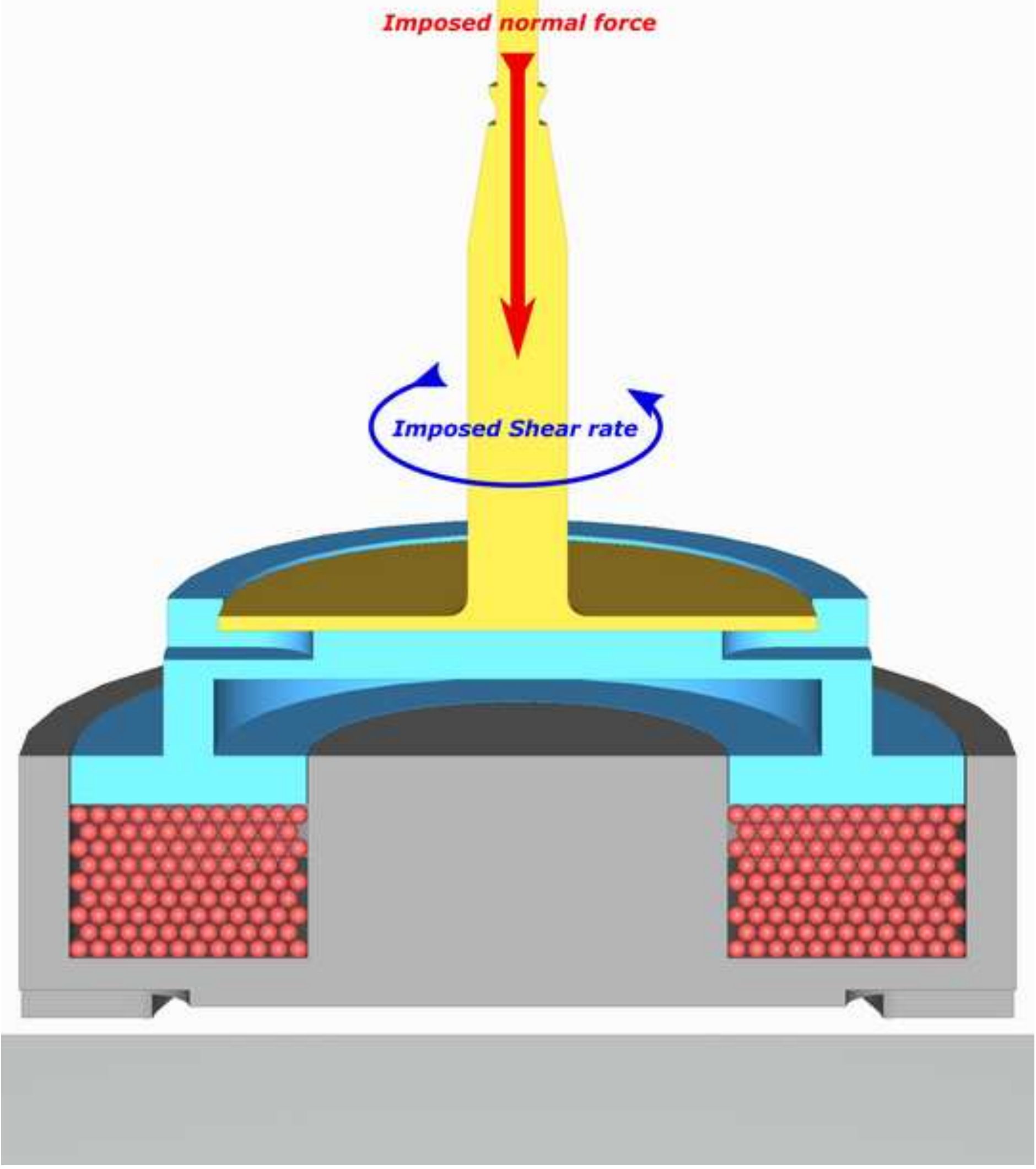


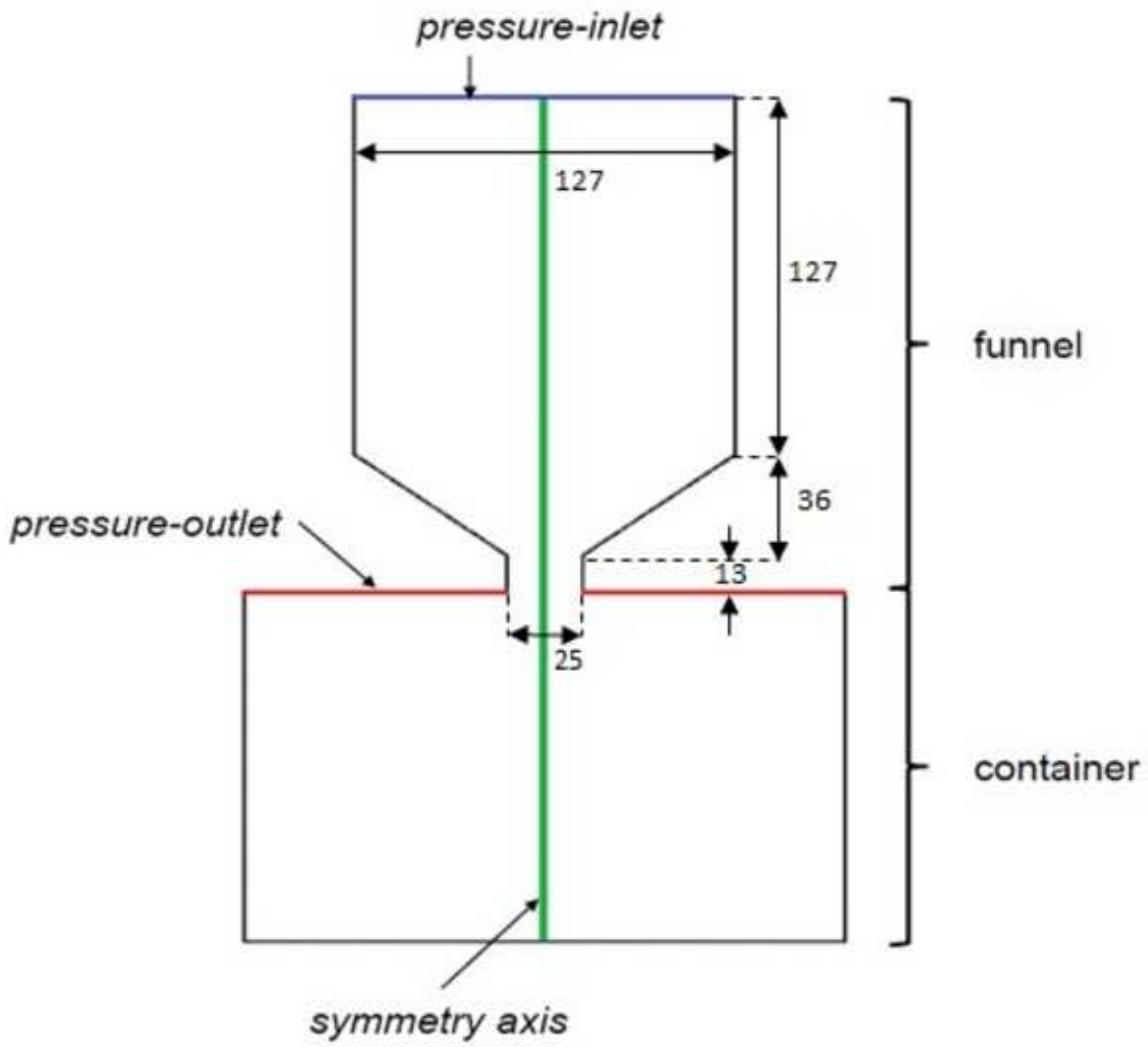


Figure 2 bottom
Click here to download high resolution image

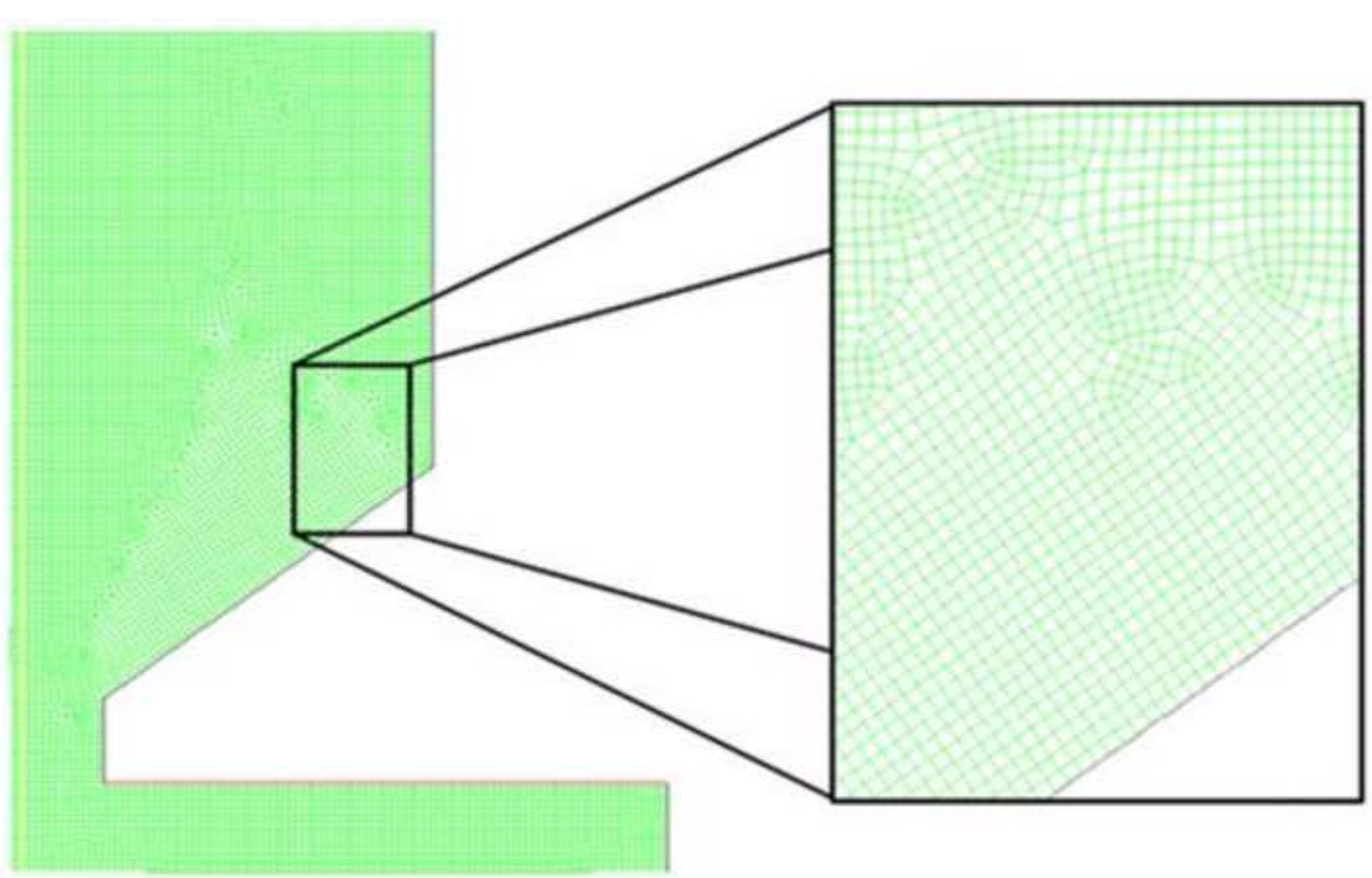

Click here to download high resolution image 
Click here to download high resolution image

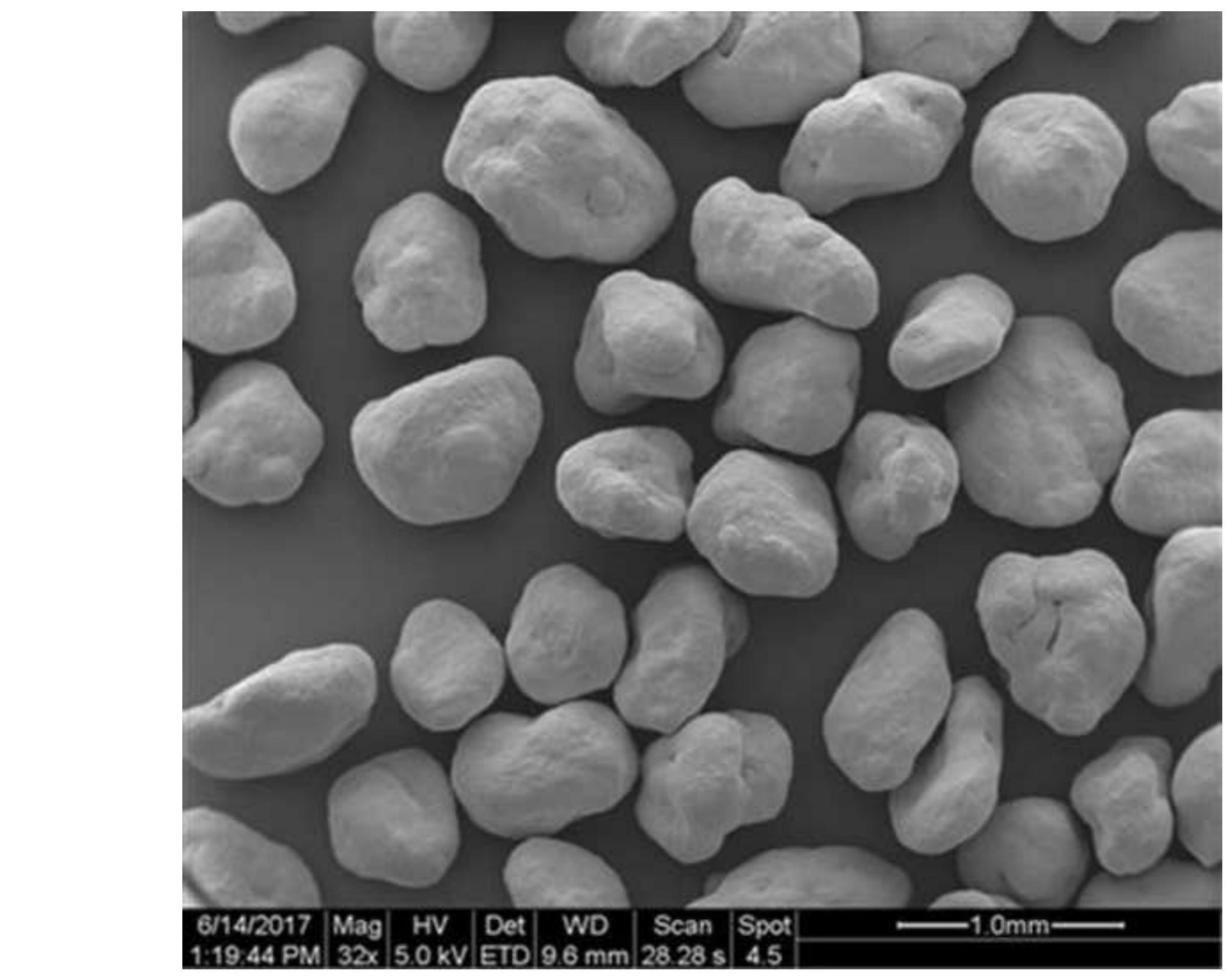

to download hich rocolution imaon

\section{bes}
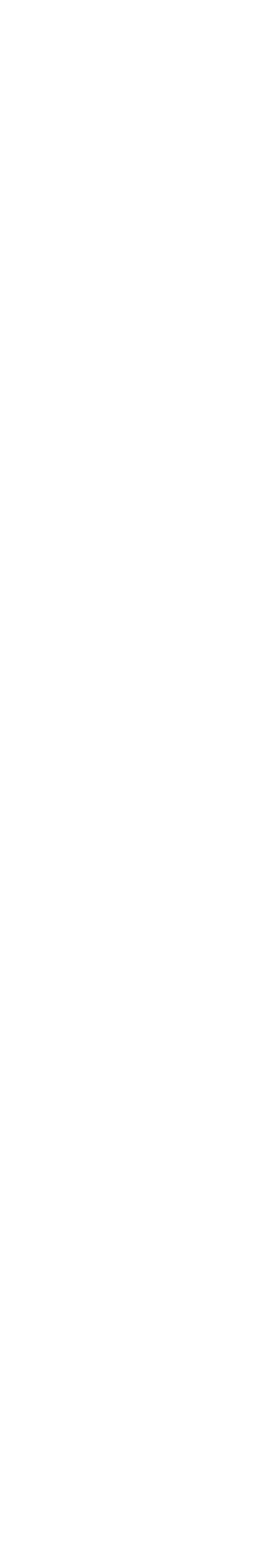
Click here to download high resolution image

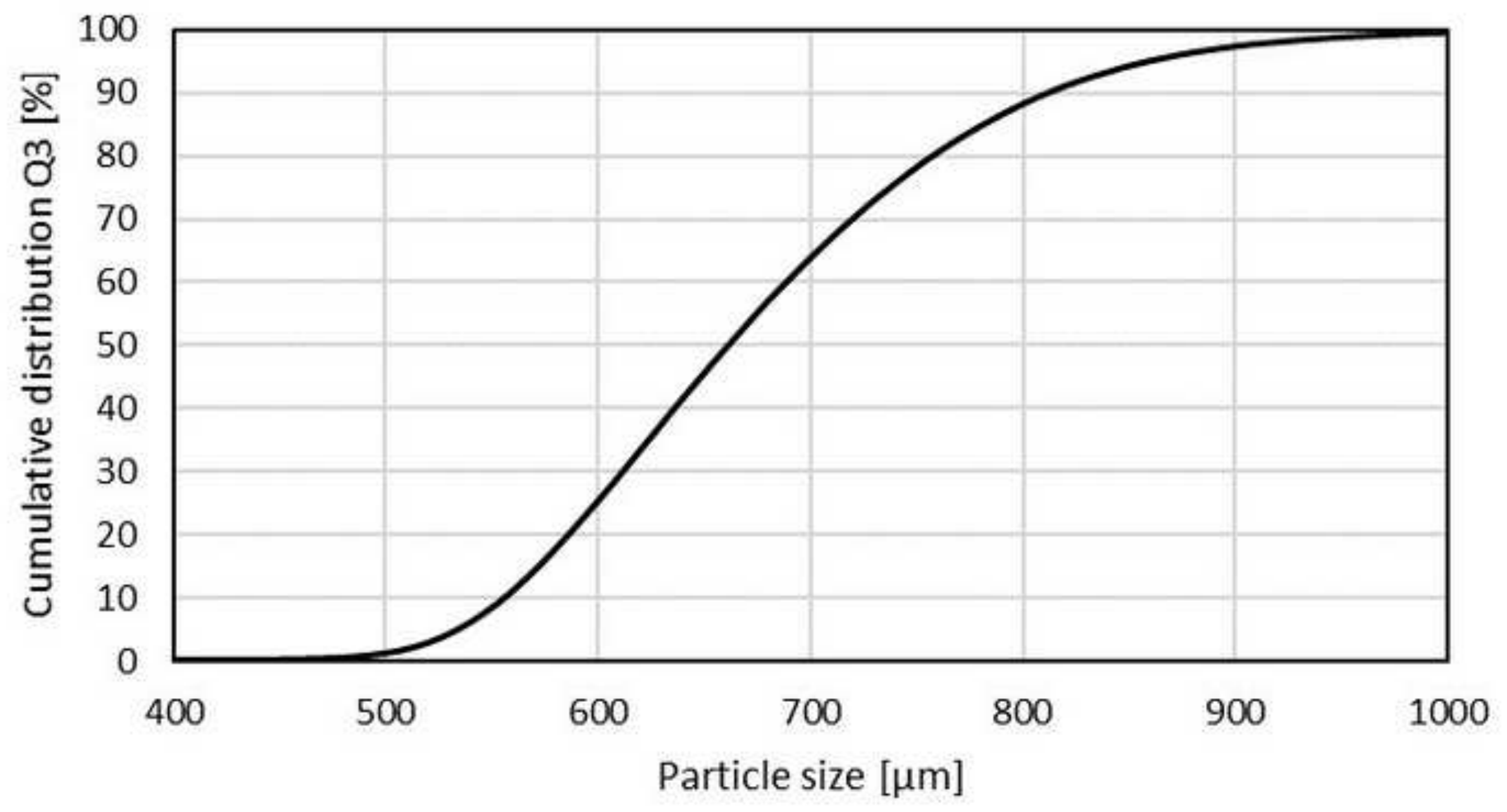




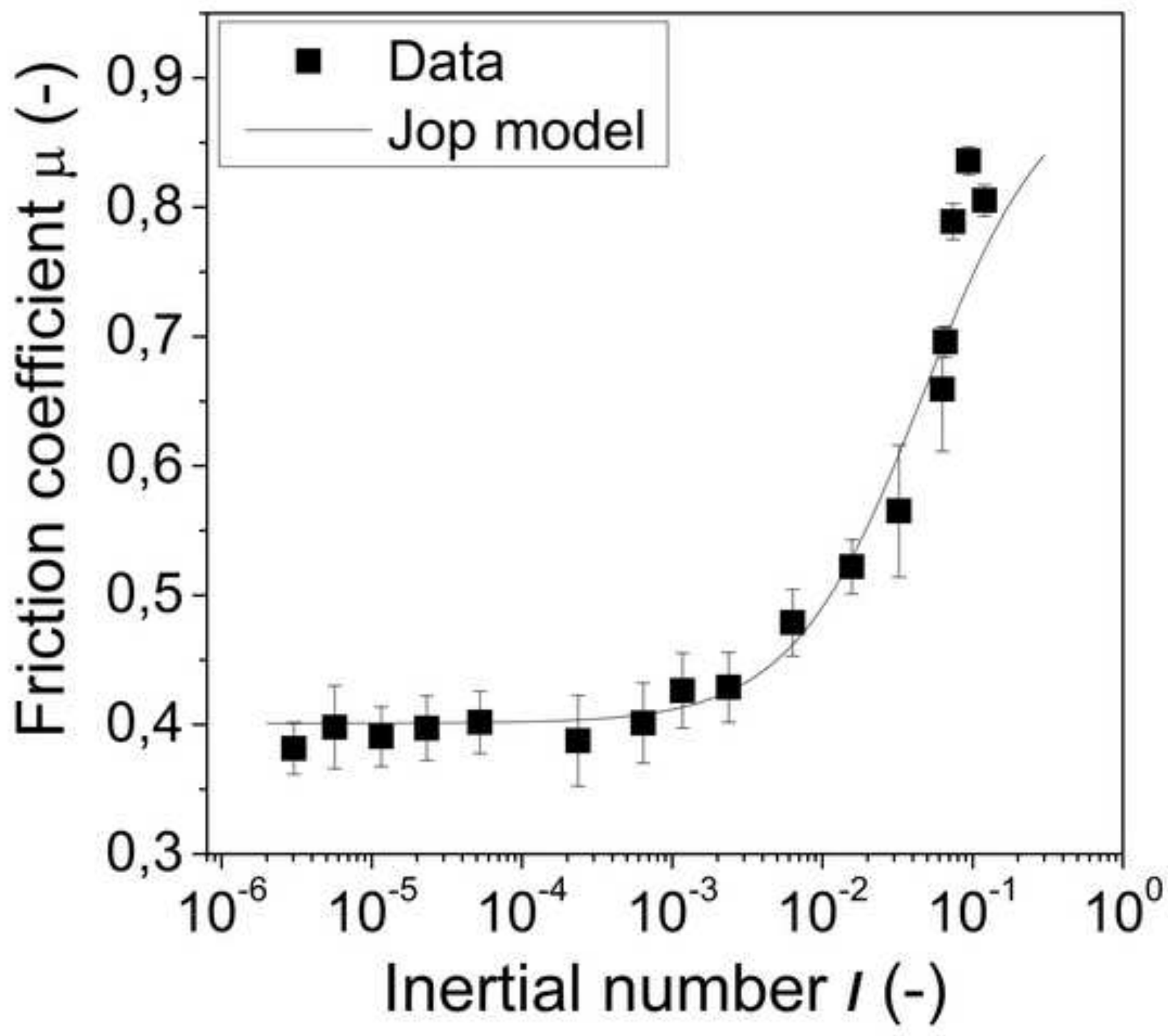




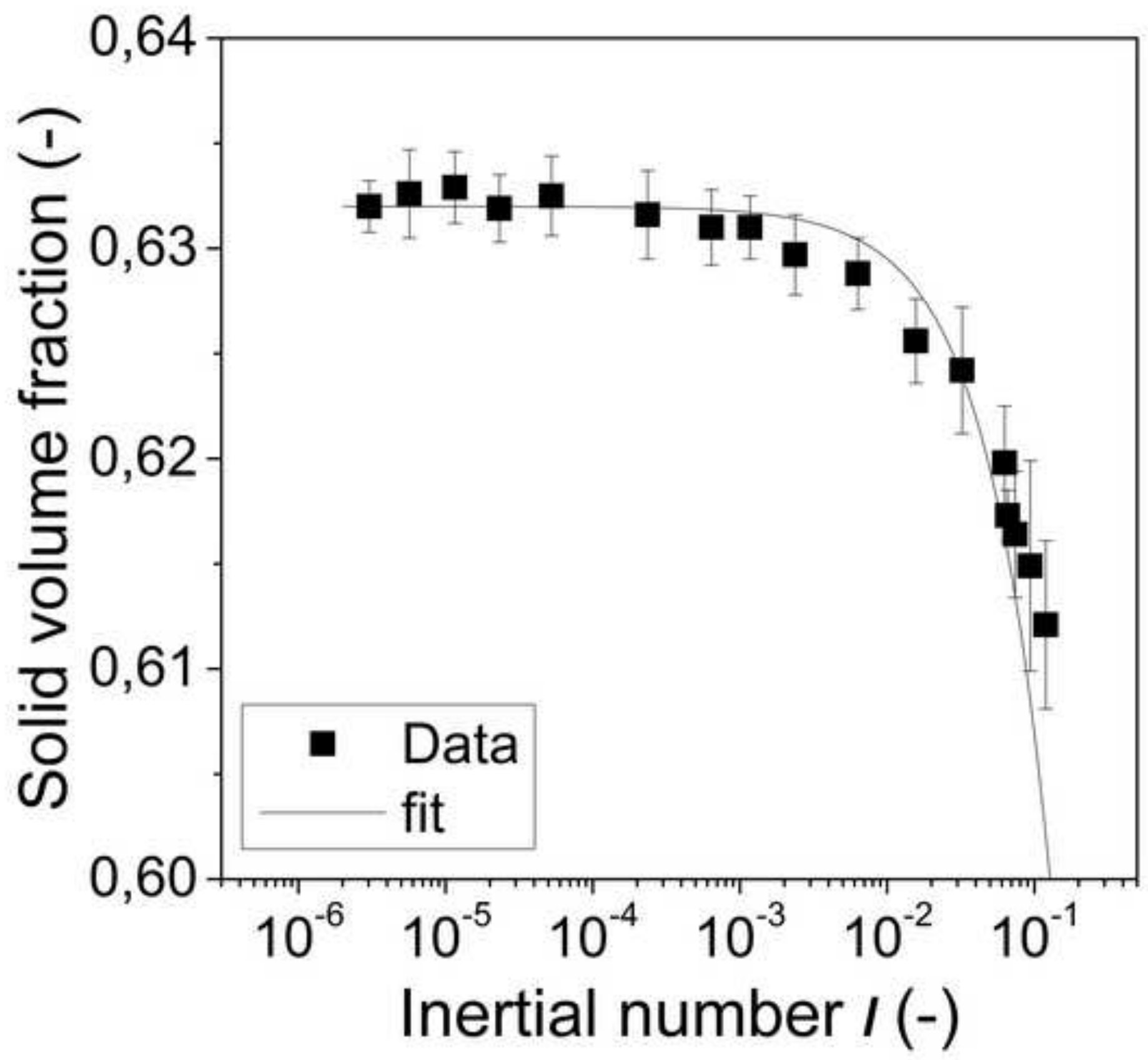




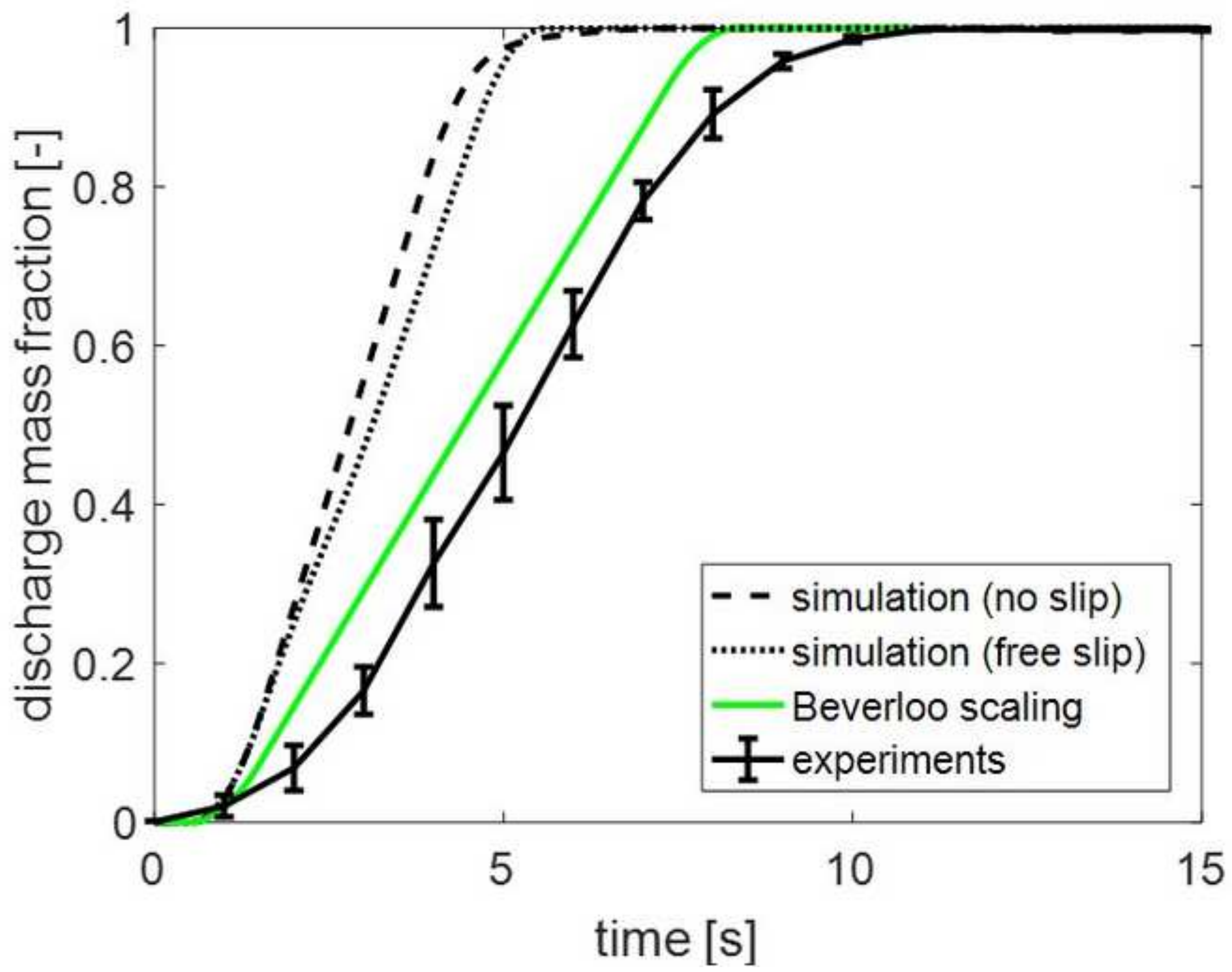




$$
t=0.5 \mathrm{~s}
$$$$
t=2 \mathrm{~s}
$$$$
t=4 \mathrm{~s}
$$

\section{no slip}
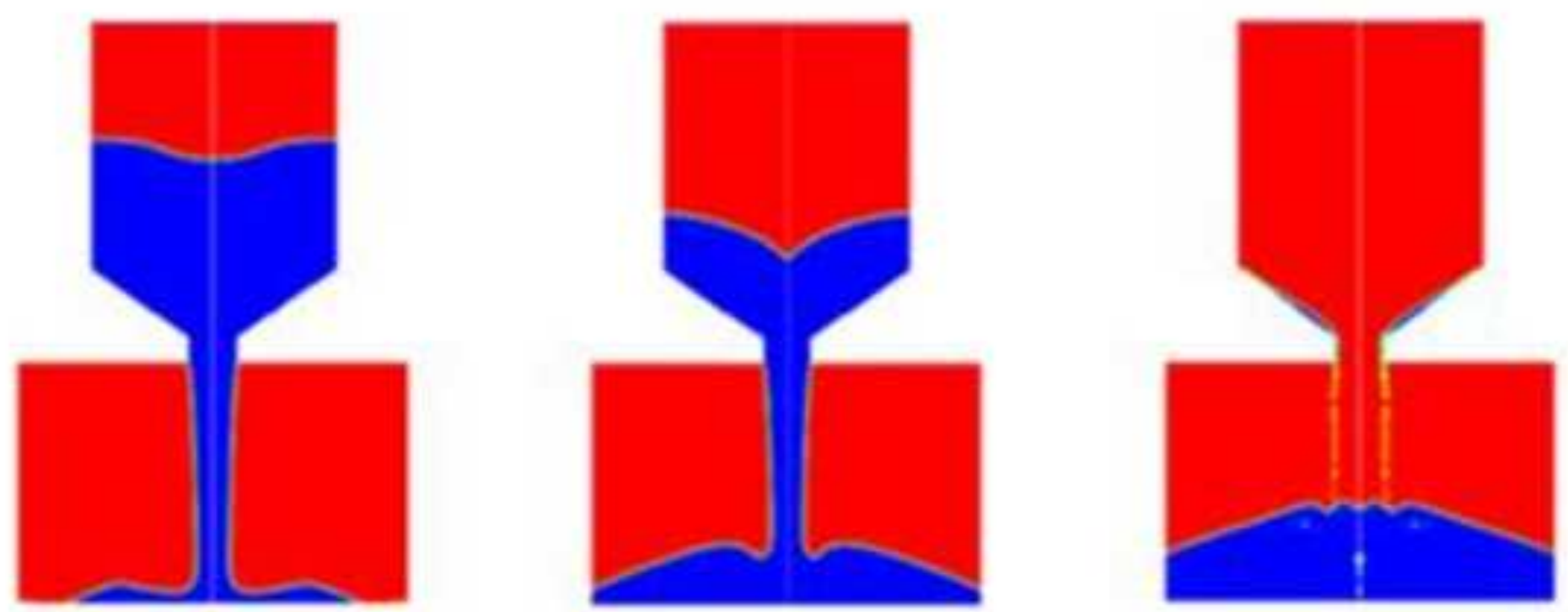

free slip
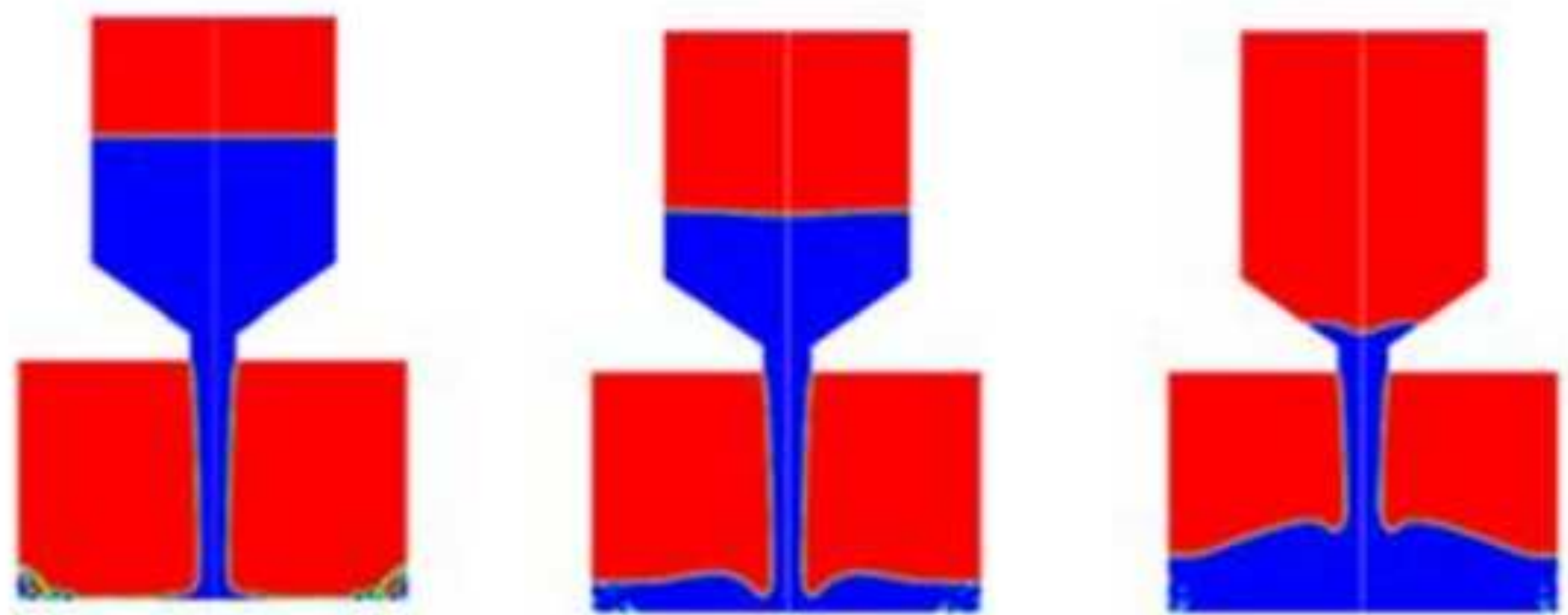


$$
t=0.5 \mathrm{~s} \quad t=1 \mathrm{~s} \quad t=2 \mathrm{~s} \quad t=3 \mathrm{~s} \quad t=4 \mathrm{~s}
$$
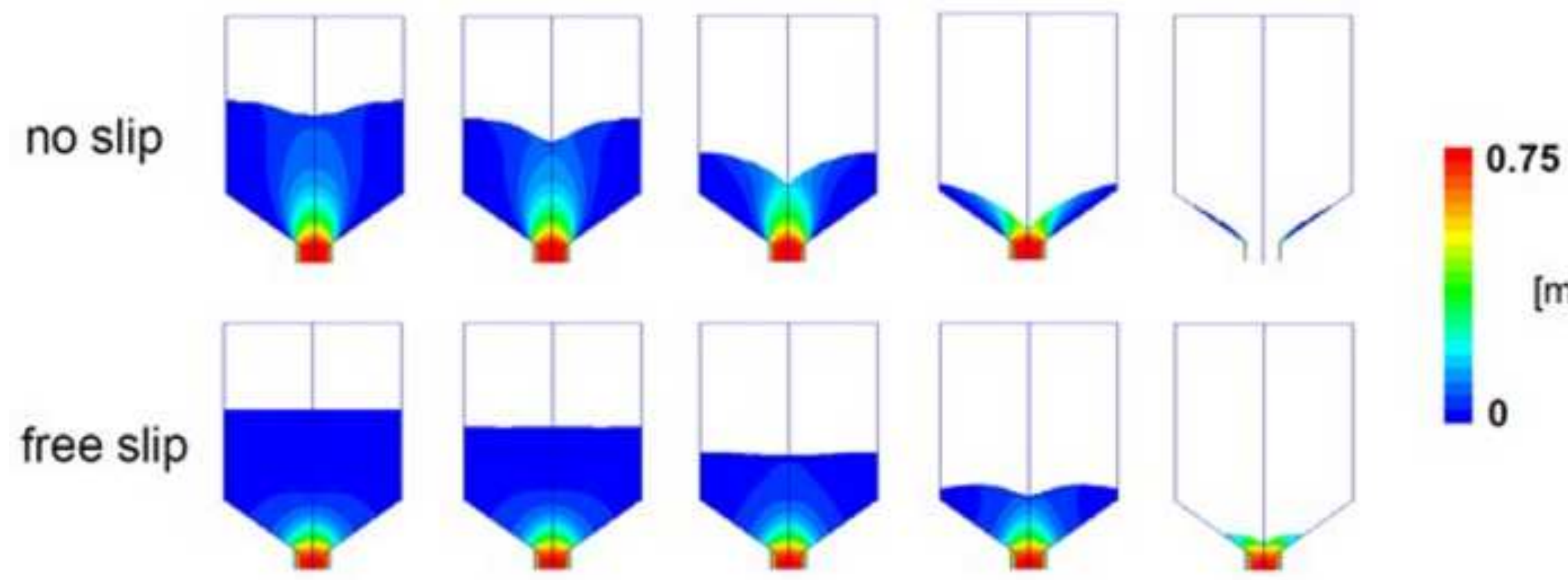

$[\mathrm{m} / \mathrm{s}]$ 
$t=0.5 \mathrm{~s}$

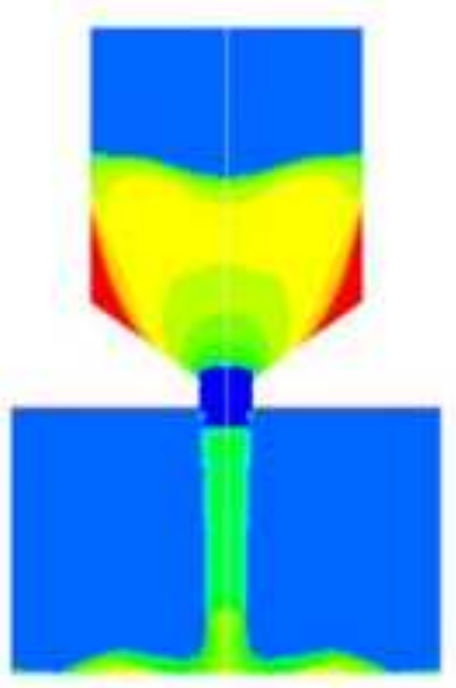

$t=1 \mathrm{~s}$

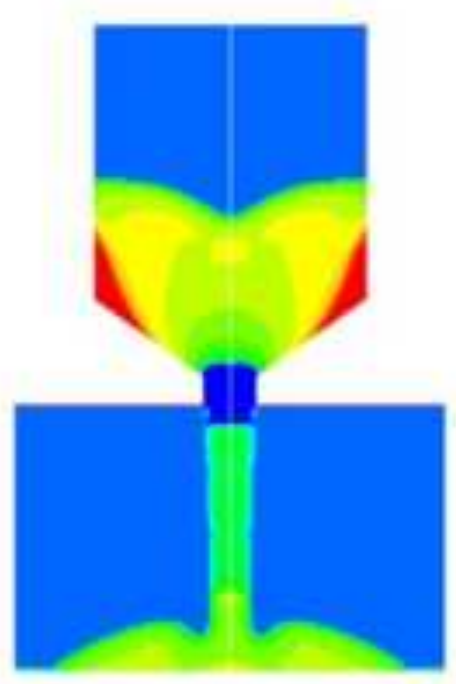

$t=2 \mathrm{~s}$

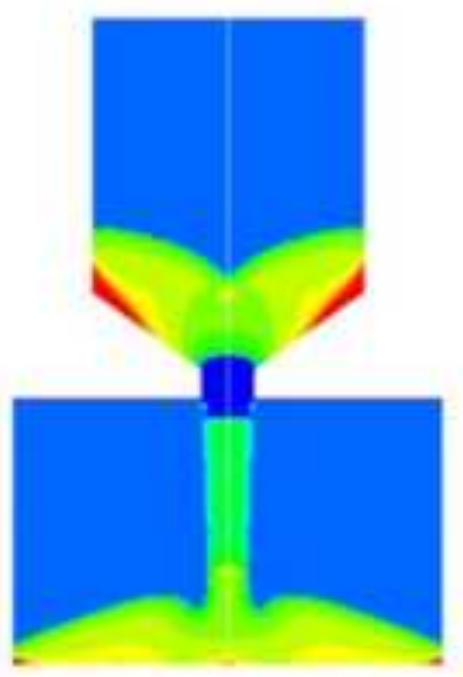

$t=3 \mathrm{~s}$

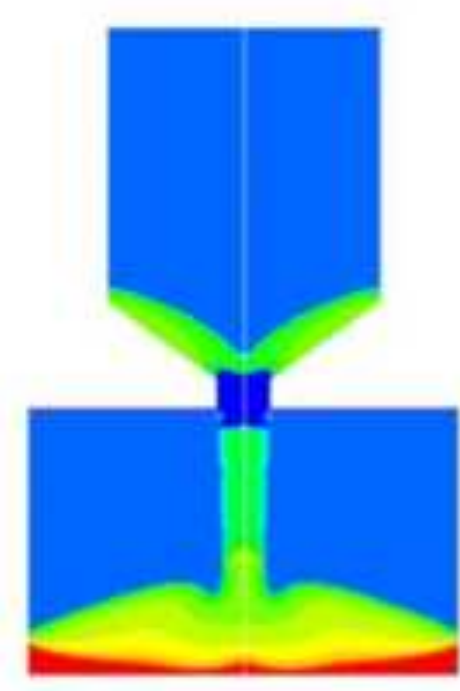

$\eta_{\max }$

0 
$t=0.5 \mathrm{~s}$

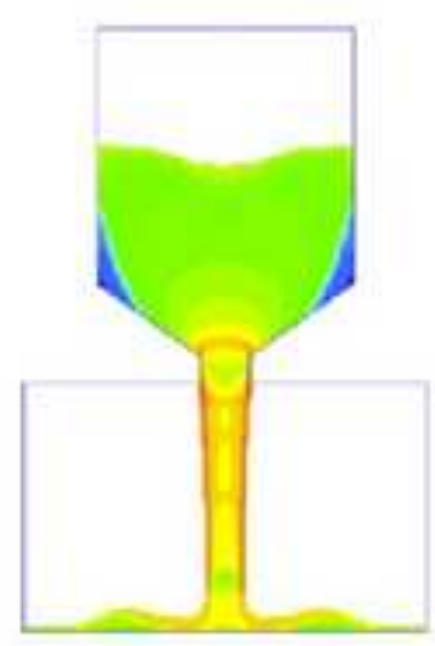

$t=1 \mathrm{~s}$

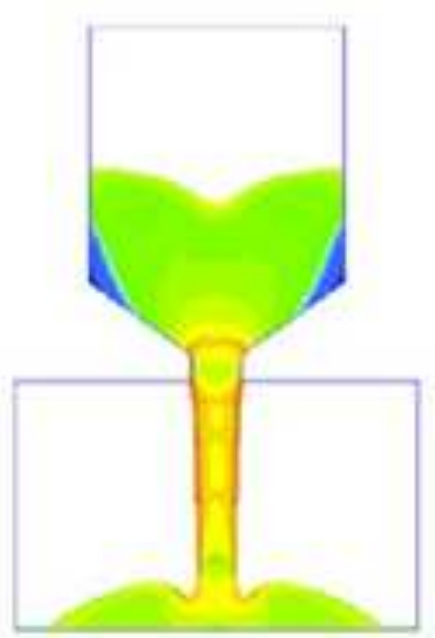

$t=2 \mathrm{~s}$

$t=3 \mathrm{~s}$

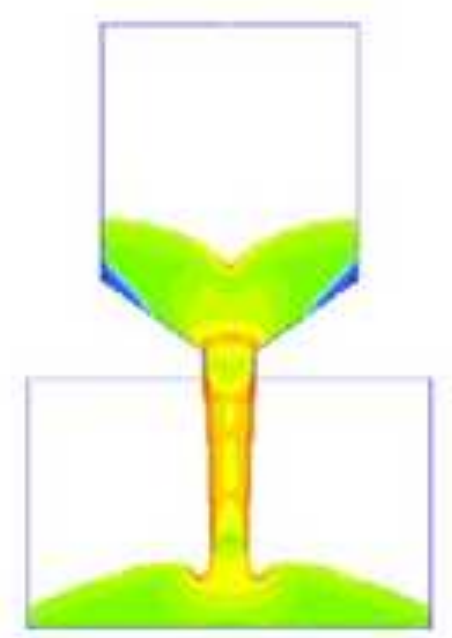

400

4

$4 \cdot 10^{-3}$

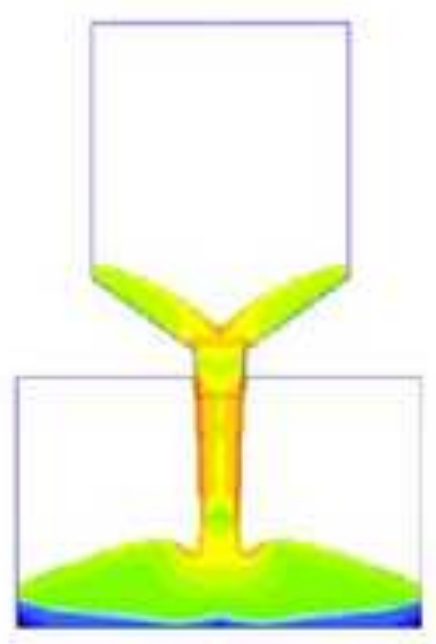

$4 \cdot 10^{-8}$ 


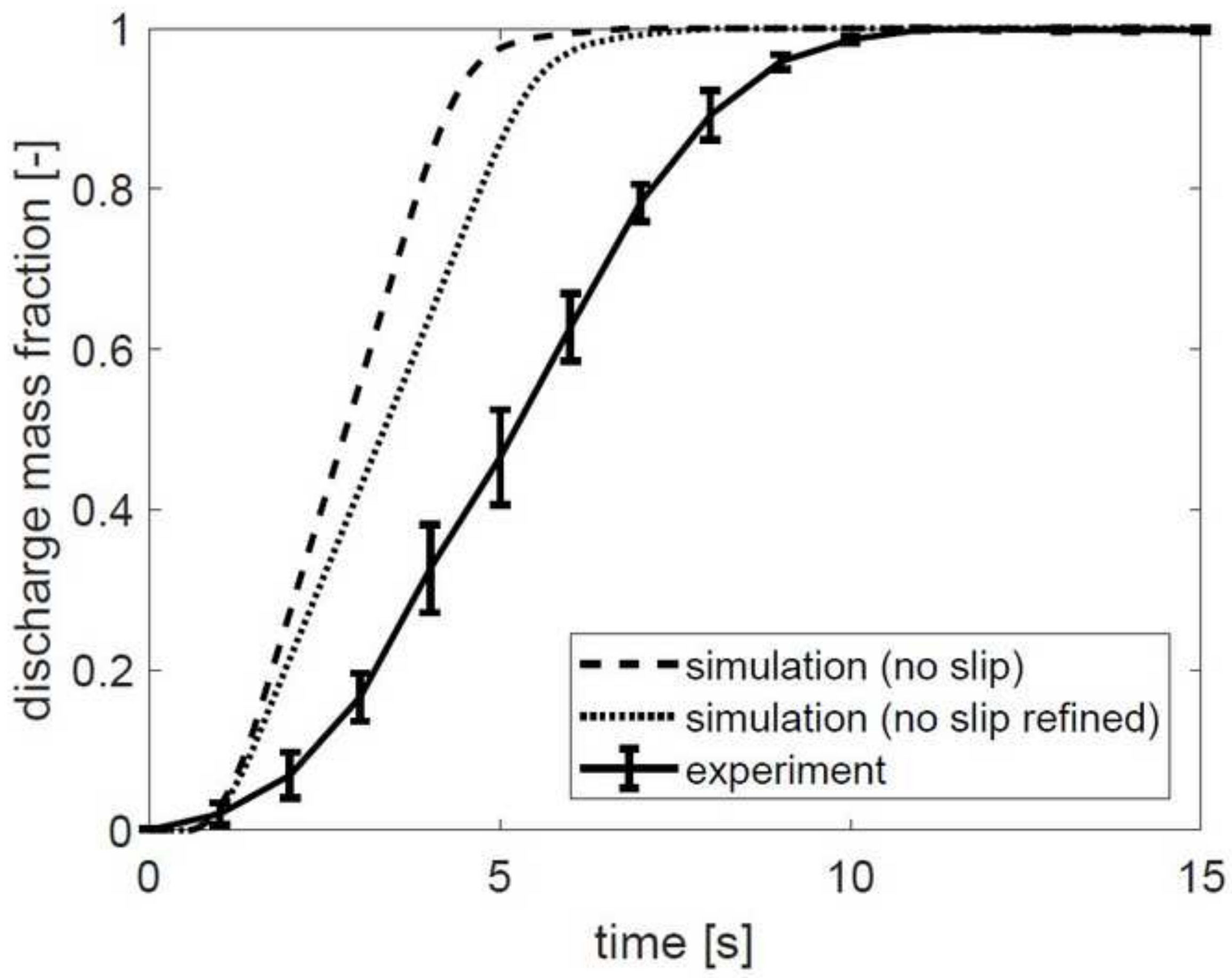


Table 1. The measured and predicted discharge rate during the steady phase.

\begin{tabular}{|l|l|}
\hline Case & Discharge rate during the steady phase $[\mathrm{g} / \mathrm{s}]$ \\
\hline Experiment & 161 \\
\hline Beverloo, $k=1$ & 151 \\
\hline Beverloo, $k=3$ & 131 \\
\hline Simulation, free-slip & 253 \\
\hline Simulation, no-slip & 302 \\
\hline Simulation, no-slip refined & 222 \\
\hline
\end{tabular}


Supplementary Material

Click here to download Supplementary Material: Discharge_VIVAPUR_MCC_Spheres_500.mp4

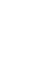

